MATHEMATICS OF COMPUTATION

Volume 78, Number 267, July 2009, Pages 1269-1292

S $0025-5718(09) 02221-2$

Article electronically published on February 18, 2009

\title{
CONVERGENT DISCRETIZATION OF HEAT AND WAVE MAP FLOWS TO SPHERES USING APPROXIMATE DISCRETE LAGRANGE MULTIPLIERS
}

\author{
SÖREN BARTELS, CHRISTIAN LUBICH, AND ANDREAS PROHL
}

\begin{abstract}
We propose fully discrete schemes to approximate the harmonic map heat flow and wave maps into spheres. The finite-element based schemes preserve a unit length constraint at the nodes by means of approximate discrete Lagrange multipliers, satisfy a discrete energy law, and iterates are shown to converge to weak solutions of the continuous problem. Comparative computational studies are included to motivate finite-time blow-up behavior in both cases.
\end{abstract}

\section{INTRODUCTION}

Let $\Omega \subset \mathbb{R}^{d}$ (for $d \geq 1$ ) be a bounded domain, and $\mathbb{S}^{m-1} \subset \mathbb{R}^{m}$ (for $m \geq 2$ ) the unit sphere. The energy of a map $\mathbf{w}: \Omega \rightarrow \mathbb{S}^{m-1}$ is defined as

$$
E(\mathbf{w})=\frac{1}{2} \int_{\Omega}|\nabla \mathbf{w}|^{2} \mathrm{~d} \mathbf{x} .
$$

Critical points are called weakly harmonic maps into the sphere 22, which are of interest in more extended models in micromagnetics [17, liquid crystal theory [1, color image denoising [24, 25, 27, 5, 6], or (in generalized form) in general relativity 20]. Related prototype nonstationary problems for solutions $\mathbf{u}: \Omega_{T} \rightarrow \mathbb{S}^{m-1}$ are: (A) the $L^{2}$-gradient flow for (1.1),

$$
\begin{array}{ll}
\mathbf{u}_{t}-\Delta \mathbf{u}=|\nabla \mathbf{u}|^{2} \mathbf{u} & \text { in } \Omega_{T}:=(0, T) \times \Omega, \\
\frac{\partial \mathbf{u}}{\partial \mathbf{n}}=0 & \text { on } \partial \Omega_{T}:=(0, T) \times \partial \Omega, \\
\mathbf{u}(0, \cdot)=\mathbf{u}_{0} & \text { in } \Omega,
\end{array}
$$

and (B) the wave map flow into the sphere $\mathbb{S}^{m-1}$,

$$
\begin{array}{ll}
\mathbf{u}_{t t}-\Delta \mathbf{u}=\left(|\nabla \mathbf{u}|^{2}-\left|\mathbf{u}_{t}\right|^{2}\right) \mathbf{u} & \text { in } \Omega_{T}, \\
\frac{\partial \mathbf{u}}{\partial \mathbf{n}}=0 & \text { on } \partial \Omega_{T}, \\
\mathbf{u}(0, \cdot)=\mathbf{u}_{0}, \quad \partial_{t} \mathbf{u}(0, \cdot)=\mathbf{v}_{0} & \text { in } \Omega .
\end{array}
$$

In both cases, static solutions are harmonic maps to the sphere; clearly, evolution is different in (A) and (B). For problem (A), existence of weak solutions can be found

Received by the editor April 10, 2007 and, in revised form, April 30, 2008.

2000 Mathematics Subject Classification. Primary 65M12, 65M60, 35K55, 35Q35.

(C)2009 American Mathematical Society Reverts to public domain 28 years from publication 
in 12]; the development of singularities in finite time, i.e., $\lim \sup _{t \rightarrow T^{-}}\|\mathbf{u}(t, \cdot)\|_{W^{1, \infty}}$ $=\infty$, is shown in [13, 11] for equivariant initial data. As to problem (B), (stable self-similar) finite-time blow-up behavior of existing weak solutions (cf. [19, 9, 26]) is known in the $(3+1)$-dimensional case using equivariant initial data, and existence of $(k$-)equivariant solutions (with winding number $k \geq 4)$ in a $(2+1)$-dimensional Minkowski space-time setting is known, which lead to finite-time blow-up [18, 10].

Numerical analysis of problems (A) and (B) is nontrivial for the following reasons:

1) In order to approximate (or construct in the limit) weak solutions in both cases (A) and (B) by using fully practical schemes based on finite elements, we cannot benefit from regularity properties of solutions in both cases, but we need to verify crucial stability properties, like discrete sphere constraint and discrete energy identity.

2) Straightforward spatio-temporal discretizations, together with standard finite elements violate the sphere constraint, and lack a discrete energy law; see e.g. [3, 15.

3) Convergent penalization strategies of problems (A) and (B) which use a Ginzburg-Landau penalty (with parameter $\varepsilon>0$ ) to approximate the sphere constraint allow for convergent discretizations for every $\varepsilon>0$; however, to specify this parameter in terms of discretization parameters is a nontrivial task, in particular, in the context of blow-up behavior of weak solutions to these problems; cf. [7] for computational evidence for problem (B).

In [3, 4, a fully practical implicit scheme is given to solve problem (A), which is based on a reformulation of (1.2) using cross products $(m=3)$,

$$
\mathbf{u}_{t}+\mathbf{u} \times(\mathbf{u} \times \Delta \mathbf{u})=0 \quad \text { in } \Omega_{T} .
$$

Together with reduced spatial integration, midpoint formula, as well as projected discrete Laplacian, a lowest order conforming finite element discretization enjoys a discrete sphere constraint and energy law, and solutions unconditionally (sub-)converge to weak solutions; moreover, a simple fixed point strategy is proposed to successively solve linear problems, whose solutions still satisfy the discrete sphere constraint, and conditionally converge to weak solutions by a contraction argument. Unfortunately, this strategy is not successful for problem (B); instead, an explicit time-splitting scheme was proposed for problem (B) in [7, and conditional convergence towards weak solutions is verified; here the idea, which originally was given in 2 in a different context, is to discretize the following reformulation of (1.5), which uses special test-functions $\mathbf{w} \in C\left([0, T) \times \Omega, \mathbb{R}^{m}\right)$ which satisfy $\langle\mathbf{u}, \mathbf{w}\rangle=0$ a.e. in $\Omega_{T}$, such that

$$
\int_{0}^{T}\left(\partial_{t}^{2} \mathbf{u}, \mathbf{w}\right) \mathrm{d} t+\int_{0}^{T}(\nabla \mathbf{u}, \nabla \mathbf{w}) \mathrm{d} t=0
$$

In this work, we construct different convergent discretizations for both problems (A) and (B), which use approximate discrete Lagrange multipliers. To motivate the approach, recall that to describe the gradient flow for (1.1) requires mappings $\mathbf{u}: \Omega_{T} \rightarrow \mathbb{R}^{m}$, and a Lagrange multiplier $\lambda: \Omega_{T} \rightarrow \mathbb{R}^{+}$, such that

$$
\mathbf{u}_{t}-\Delta \mathbf{u}=\lambda \mathbf{u} \quad \text { and } \quad|\mathbf{u}|=1 \quad \text { in } \Omega_{T} .
$$


In fact, $\lambda=|\nabla \mathbf{u}|^{2}$ is easy to verify in the present case, where the target manifold is the sphere. The following scheme uses an approximate discrete Lagrange multiplier to enforce both the discrete sphere constraint, i.e., unit length of (iterates of) finite element functions at nodes of a triangulation $\mathcal{T}_{h}$ and a discrete energy law.

We employ some notation which is further detailed in Section 2, Let $V_{h} \subset$ $W^{1,2}(\Omega)$ be the lowest order conforming finite element space subordinate to a triangulation $\mathcal{T}_{h}$ of $\Omega$, and $\mathbf{V}_{h}=\left[V_{h}\right]^{m}$. By $\mathcal{N}_{h}$, we denote the set of all nodes associated with $\mathcal{T}_{h}$. Below, $(\cdot, \cdot)_{h}$ denotes the discrete version (reduced integration) of the inner product in $L^{2}\left(\Omega, \mathbb{R}^{m}\right)$, and we use $d_{t} \varphi^{n}:=k^{-1}\left(\varphi^{n}-\varphi^{n-1}\right)$, and $\varphi^{n+1 / 2}:=\frac{1}{2}\left(\varphi^{n+1}+\varphi^{n}\right)$ with $n \geq 1$, for a sequence $\left\{\varphi^{n}\right\}_{n \geq 0}$, and for an equidistant time-step size $k>0$. Then, the approximation scheme for problem (A) reads as follows.

Algorithm A. For $n \geq 0$, let $\mathbf{U}^{n} \in \mathbf{V}_{h}$ be given, and find $\left(\mathbf{U}^{n+1}, \lambda^{n+1}\right) \in \mathbf{V}_{h} \times V_{h}$, such that

$$
\begin{aligned}
& \left(d_{t} \mathbf{U}^{n+1}, \boldsymbol{\Phi}\right)_{h}+\left(\nabla \mathbf{U}^{n+1 / 2}, \nabla \boldsymbol{\Phi}\right)=\left(\lambda^{n+1} \mathbf{U}^{n+1 / 2}, \boldsymbol{\Phi}\right)_{h} \quad \forall \boldsymbol{\Phi} \in \mathbf{V}_{h}, \\
& \left|\mathbf{U}^{n+1}(\mathbf{z})\right|=1 \quad \forall \mathbf{z} \in \mathcal{N}_{h} .
\end{aligned}
$$

As we will show in Section 3, an explicit formula to compute $\lambda^{n+1}=\lambda^{n+1}\left(\mathbf{U}^{n+1 / 2}\right)$ is available; however, in contrast to the continuous Lagrange parameter above, its computation at a single node $\mathbf{z} \in \mathcal{N}_{h}$ requires to consider values of $\mathbf{U}^{n+1 / 2}$ at neighboring ones - which accounts for finite values $k, h>0$ in the discretization scheme. Conditional solvability of Algorithm A holds by Lemma 3.1. The method is devised such that a discrete energy identity holds: choose $\boldsymbol{\Phi}=d_{t} \mathbf{U}^{n+1}$ in (1.8), and use reduced integration for the first and last term, together with $\left|\mathbf{U}^{n+1}(\mathbf{z})\right|^{2}-\left|\mathbf{U}^{n}(\mathbf{z})\right|^{2}=0$, for all $\mathbf{z} \in \mathcal{N}_{h}$, to obtain

$$
\left\|d_{t} \mathbf{U}^{n+1}\right\|_{h}^{2}+\left(\nabla \mathbf{U}^{n+1 / 2}, \nabla d_{t} \mathbf{U}^{n+1}\right)=0,
$$

and after summation over all iteration steps $0 \leq n \leq N$,

$$
E\left(\mathbf{U}^{N+1}\right)+k \sum_{n=0}^{N}\left\|d_{t} \mathbf{U}^{n+1}\right\|_{h}^{2}=E\left(\mathbf{U}^{0}\right) .
$$

As is worked out in Section [3, this discrete energy law is then crucial to verify (subsequence) convergence of iterates from Algorithm A to weak solutions of (1.2)(1.4); see Theorem 3.1 .

Remark 1.1. Note that $\lambda^{n+1} \in V_{h}$ (for $n \geq 0$ ) is not the Lagrange multiplier associated to the discrete sphere constraint $\left|\mathbf{U}^{n+1}(\mathbf{z})\right|^{2}=1$ for all $\mathbf{z} \in \mathcal{N}_{h}$, since the right-hand side of (1.8) is modified from $\left(\lambda^{n+1} \mathbf{U}^{n+1}, \boldsymbol{\Phi}\right)_{h}$ to $\left(\lambda^{n+1} \mathbf{U}^{n+1 / 2}, \boldsymbol{\Phi}\right)_{h}$ to obtain the discrete energy law (1.10).

A similar program is now evident for the wave map problem (1.5)-(1.7), where (1.5) is of the form

$$
\mathbf{u}_{t t}-\Delta \mathbf{u}=\lambda \mathbf{u} \quad \text { and } \quad|\mathbf{u}|=1 \quad \text { in } \Omega_{T},
$$

with $\lambda=\left(|\nabla \mathbf{u}|^{2}-\left|\mathbf{u}_{t}\right|^{2}\right)$ in the present form. In Section 4, we show conditional convergence of the following implicit discretization, which again uses approximate discrete Lagrange multipliers. 
Algorithm B. For $n \geq 0$, let $\mathbf{U}^{n}, \mathbf{U}^{n-1} \in \mathbf{V}_{h}$ be given, and find $\left(\mathbf{U}^{n+1}, \lambda^{n+1}\right) \in$ $\mathbf{V}_{h} \times V_{h}$, such that

$$
\begin{aligned}
& \left(d_{t}^{2} \mathbf{U}^{n+1}, \boldsymbol{\Phi}\right)_{h}+\left(\nabla \mathbf{U}^{n+1 / 2}, \nabla \boldsymbol{\Phi}\right)=\left(\lambda^{n+1} \mathbf{U}^{n+1 / 2}, \boldsymbol{\Phi}\right)_{h} \quad \forall \boldsymbol{\Phi} \in \mathbf{V}_{h}, \\
& \left|\mathbf{U}^{n+1}(\mathbf{z})\right|=1 \quad \forall \mathbf{z} \in \mathcal{N}_{h} .
\end{aligned}
$$

For given initial velocity $\mathbf{V}^{0}$, the second starting value is chosen as $\mathbf{U}^{-1}=$ $\mathbf{U}^{0}-k \mathbf{V}^{0}$. This value does not satisfy the sphere constraint, but it is used only for starting the recurrence relation of the algorithm.

As for problem (A), a discrete energy law can be shown, using $E_{h}(\mathbf{V}, \mathbf{U})=$ $\frac{1}{2}\left[\|\mathbf{V}\|_{h}^{2}+\|\nabla \mathbf{U}\|^{2}\right]$,

$$
E_{h}\left(d_{t} \mathbf{U}^{N+1}, \mathbf{U}^{N+1}\right)+\frac{k^{2}}{2} \sum_{n=0}^{N}\left\|d_{t}^{2} \mathbf{U}^{n+1}\right\|_{2}^{2}=E_{h}\left(\mathbf{V}^{0}, \mathbf{U}^{0}\right) \quad(N \geq 1)
$$

and conditional solvability; cf. Lemma 4.1. As is evident from the second term in (1.13), Algorithm B uses numerical dissipation, while a symmetric and conservative discretization of (1.5)-(1.7), which replaces (1.11) by

$$
\left(d_{t}^{2} \mathbf{U}^{n+1}, \boldsymbol{\Phi}\right)_{h}+\left(\frac{1}{2} \nabla\left[\mathbf{U}^{n+1}+\mathbf{U}^{n-1}\right], \nabla \boldsymbol{\Phi}\right)=\left(\frac{\lambda^{n}}{2}\left[\mathbf{U}^{n+1}+\mathbf{U}^{n-1}\right], \boldsymbol{\Phi}\right)_{h},
$$

is discussed in Remark 4.1. However, in the analysis for Algorithm B in Section 4. its dissipative character is needed to conclude convergence of iterates towards weak solutions of (1.5)-(1.7); see Theorem 4.1.

The main result for the heat flow of harmonic maps is Theorem 3.1, which verifies subsequence convergence of iterates from the implicit Algorithm A towards weak solutions of problem (A), provided that $k \leq C h^{2}$; this (unexpected) mesh constraint is sufficient for solvability for finite $(k, h)>0$, whereas both, the discrete energy law and the sphere constraint do not require mesh constraints. We remark that no constraint is required for a different discretization of (1.2)-(1.4) in [3] for the special case where the target is the two-dimensional sphere. For the wave-map equation, conditional convergence towards weak solutions of problem (B) is verified in Theorem 4.1, again, only Brouwer's fixed-point argument to verify existence of iterates requires a mesh-constraint $k \leq C h^{\max \{d / 2,1\}}$, while a discrete energy law and the sphere constraint hold unconditionally. For comparison, to validate a (slightly perturbed) discrete energy law and eventually conclude convergence for the splitting-based algorithm in [7] requires the more restrictive mesh-constraint $k=o\left(h^{\frac{4+d}{3}}\right)$ to hold. Interestingly, the fixed point iterations employed to solve the nonlinear systems of equations in the numerical experiments reported in Section 5 seem to converge exactly under these constraints, which indicates that our results may be sharp.

The remainder is organized as follows: Section 2 collects some notations which are used throughout the paper. Section 3 verifies convergence of Algorithm A to obtain weak solutions of problem $(\mathrm{A})$ in the limit $(k, h) \rightarrow 0$. Section 4 correspondingly shows convergence of Algorithm B towards problem (B). Computational experiments to motivate possible blow-up for both problems are reported in Section [5] and are compared with corresponding studies in [3] (harmonic map heat flow), and [7] (wave map equation). 


\section{Preliminaries}

Standard notation is adopted throughout this paper: $\langle\cdot, \cdot\rangle$ denotes the standard inner product of the Euclidean space $\mathbb{R}^{d},(\cdot, \cdot):=(\cdot, \cdot)_{\Omega}$ is the standard $L^{2}$-inner product over the domain $\Omega \subset \mathbb{R}^{d}$. $W^{\ell, p}\left(\Omega, \mathbb{R}^{m}\right)$ denotes the $(\ell, p)$-Sobolev space of vector-valued functions, and $\|\cdot\|_{W^{\ell, p}}$ its norm. Throughout this paper, $C>0$ is used as a generic positive $(k, h)$-independent constant which may take different values at different locations. We also introduce $u_{t}:=\partial_{t} u, \nabla u:=\left(\partial_{x_{1}} u, . ., \partial_{x_{d}} u\right)$, $D:=\left(\partial_{t}, \nabla\right)$, and define the nonlinear Sobolev space

$$
W^{1,2}\left(\Omega, \mathbb{S}^{m-1}\right)=\left\{\mathbf{u} \in W^{1,2}\left(\Omega, \mathbb{R}^{m}\right) ; \mathbf{u} \in \mathbb{S}^{m-1} \text { a.e. in } \Omega\right\},
$$

where boldface letters are used for vector-valued quantities.

For simplicity, let $\Omega$ be a bounded polygonal (when $d=2$ ) or polyhedral (when $d=3$ ) domain. Let $\mathcal{T}_{h}$ denote a quasiuniform triangulation of $\Omega$ into triangles or tetrahedra with mesh size $h>0$ for $n=2$ or $n=3$, respectively. For a domain $K \subset \mathbb{R}^{d}$, let $\mathcal{P}_{1}(K)$ stand for the set of all affine functions on $K$. We define the Lagrange finite element spaces

$$
V_{h}:=\left\{w \in C(\bar{\Omega}) ;\left.w\right|_{K} \in \mathcal{P}_{1}(K) \forall K \in \mathcal{T}_{h}\right\}, \quad \mathbf{V}_{h}:=\left[V_{h}\right]^{m} .
$$

Let $\mathcal{N}_{h}$ denote the set of all nodes associated with the finite element space $V_{h}$, and $\left\{\varphi_{\mathbf{z}} ; \mathbf{z} \in \mathcal{N}_{h}\right\}$ the nodal basis for $V_{h}$; we define the following nodal interpolation operator $\mathcal{I}_{h}: C(\bar{\Omega}) \rightarrow V_{h}$ by

$$
\mathcal{I}_{h} w:=\sum_{\mathbf{z} \in \mathcal{N}_{h}} w(\mathbf{z}) \varphi_{\mathbf{z}} \quad \forall w \in C(\bar{\Omega}) .
$$

For any two functions $\mathbf{v}, \mathbf{w} \in C\left(\bar{\Omega}, \mathbb{R}^{m}\right)$, we define a discrete $L^{2}$-inner product by

$$
(\mathbf{v}, \mathbf{w})_{h}:=\int_{\Omega} \mathcal{I}_{h}(\langle\mathbf{v}, \mathbf{w}\rangle) \mathrm{d} \mathbf{x}=\sum_{\mathbf{z} \in \mathcal{N}_{h}} \beta_{\mathbf{z}}\langle\mathbf{v}(\mathbf{z}), \mathbf{w}(\mathbf{z})\rangle,
$$

where $\beta_{\mathbf{z}}=\int_{\Omega} \varphi_{\mathbf{z}} \mathrm{d} \mathbf{x}$, for all $\mathbf{z} \in \mathcal{N}_{h}$. We also define $\|\mathbf{w}\|_{h}:=(\mathbf{w}, \mathbf{w})_{h}^{\frac{1}{2}}$. It is easy to check that there holds for all $\mathbf{v}_{h}, \mathbf{w}_{h} \in \mathbf{V}_{h}$,

$$
\begin{aligned}
& \left\|\mathbf{w}_{h}\right\|_{L^{2}} \leq\left\|\mathbf{w}_{h}\right\|_{h} \leq(d+2)^{\frac{1}{2}}\left\|w_{h}\right\|_{L^{2}}, \\
& \left|\left(\mathbf{v}_{h}, \mathbf{w}_{h}\right)_{h}-\left(\mathbf{v}_{h}, \mathbf{w}_{h}\right)\right| \leq C h\left\|\mathbf{v}_{h}\right\|_{L^{2}}\left\|\nabla \mathbf{w}_{h}\right\|_{L^{2}} .
\end{aligned}
$$

\section{HARMONiC MAP heAT FLOW TO THE SPHERE}

We numerically approximate weak solutions of (1.2)-(1.4) in the sense of [22]. We refer to [22] and the references therein for proofs of existence of weak solutions.

Definition 3.1. Given $\mathbf{u}_{0} \in W^{1,2}\left(\Omega, \mathbb{S}^{m-1}\right)$ and $T>0$, a function $\mathbf{u} \in W^{1,2}\left(\Omega_{T}, \mathbb{R}^{m}\right)$ is called a weak solution of (1.2)-(1.4) if (i) $\mathbf{u}(0, \cdot)=\mathbf{u}_{0} \in W^{1,2}\left(\Omega, \mathbb{S}^{m-1}\right)$ in the sense of traces, (ii) $|\mathbf{u}|=1$ almost everywhere in $\Omega_{T}$, (iii) for almost all $T^{\prime} \in(0, T)$ there holds

$$
\frac{1}{2} \int_{\Omega}\left|\nabla \mathbf{u}\left(T^{\prime}, \mathbf{x}\right)\right|^{2} \mathrm{~d} \mathbf{x}+\int_{0}^{T^{\prime}}\left\|\partial_{t} \mathbf{u}(t, \cdot)\right\|_{L^{2}}^{2} \mathrm{~d} t \leq \frac{1}{2} \int_{\Omega}\left|\nabla \mathbf{u}_{0}(\mathbf{x})\right|^{2} \mathrm{~d} \mathbf{x},
$$

and (iv) for all $\phi \in C^{\infty}\left(\bar{\Omega}_{T}, \mathbb{R}^{m}\right)$ there holds

$$
\int_{\Omega_{T}}\left\langle\partial_{t} \mathbf{u}, \mathbf{u} \wedge \boldsymbol{\phi}\right\rangle \mathrm{d} \mathbf{x} \mathrm{d} t+\int_{\Omega_{T}}\langle\nabla \mathbf{u}, \nabla(\mathbf{u} \wedge \boldsymbol{\phi})\rangle \mathrm{d} \mathbf{x} \mathrm{d} t=0 .
$$


For $m=3$ here we have the usual wedge or cross product in $\mathbb{R}^{3}$. For $m>3$, an expression $\langle\mathbf{u}, \mathbf{v} \wedge \mathbf{w}\rangle$ is to be interpreted as the 3 -volume of the parallelepiped in the vector space spanned by $\mathbf{u}, \mathbf{v}, \mathbf{w}$. It turns out that the usual differentiation rules for $\langle\mathbf{u}, \mathbf{v} \wedge \mathbf{w}\rangle$ as for the cross product in $\mathbb{R}^{3}$ remain valid also in the higherdimensional case. The case $m=2$ is also covered by taking $\phi$ as a scalar-valued function and interpreting $\langle\mathbf{u}, \mathbf{v} \wedge \phi\rangle=\left\langle\mathbf{u}, \mathbf{v}^{\perp} \phi\right\rangle$ as $\phi$ times the oriented area of the parallelogram spanned by $\mathbf{u}$ and $\mathbf{v}$.

We recapitulate Algorithm A where the approximate discrete Lagrange multiplier $\lambda^{n+1}$ is now specified explicitly. This is obtained by choosing $\boldsymbol{\Phi}=\mathbf{U}^{n+1 / 2}(\mathbf{z}) \varphi_{\mathbf{z}}$ in (1.8) and noting that then $\left(d_{t} \mathbf{U}^{n+1}, \boldsymbol{\Phi}\right)_{h}=\beta_{\mathbf{z}}\left(\left|\mathbf{U}^{n+1}(\mathbf{z})\right|^{2}-\left|\mathbf{U}^{n}(\mathbf{z})\right|^{2}\right) / 2 k$, which should be zero for all $\mathbf{z} \in \mathcal{N}_{h}$. We use the expression for $\lambda^{n+1}$ both, in the implementation of the algorithm by a fixed-point iteration and to study necessary conditions for well-posedness for finite $(k, h)$ and convergence behavior for $(k, h) \rightarrow 0$.

Algorithm A. Let $\mathbf{U}^{0} \in \mathbf{V}_{h}$, with $\left|\mathbf{U}^{0}(\mathbf{z})\right|=1$ for all $\mathbf{z} \in \mathcal{N}_{h}$. For $n=0,1,2, \ldots$, find $\left(\mathbf{U}^{n+1}, \lambda^{n+1}\right) \in \mathbf{V}_{h} \times V_{h}$, such that for all $\boldsymbol{\Phi} \in \mathbf{V}_{h}$ and all $\mathbf{z} \in \mathcal{N}_{h}$ there holds

$$
\begin{aligned}
& \left(d_{t} \mathbf{U}^{n+1}, \boldsymbol{\Phi}\right)_{h}+\left(\nabla \mathbf{U}^{n+1 / 2}, \nabla \boldsymbol{\Phi}\right)=\left(\lambda^{n+1} \mathbf{U}^{n+1 / 2}, \boldsymbol{\Phi}\right)_{h}, \\
& \lambda^{n+1}(\mathbf{z})= \begin{cases}0 & \text { if } \mathbf{U}^{n+1 / 2}(\mathbf{z})=0, \\
\frac{\left(\nabla \mathbf{U}^{n+1 / 2}, \mathbf{U}^{n+1 / 2}(\mathbf{z}) \otimes \nabla \varphi_{\mathbf{z}}\right)}{\beta_{\mathbf{z}}\left|\mathbf{U}^{n+1 / 2}(\mathbf{z})\right|^{2}} & \text { else. }\end{cases}
\end{aligned}
$$

Next, we verify solvability for Algorithm A for restricted choices $k=\mathcal{O}\left(h^{2}\right)$ and quasiuniform meshes $\mathcal{T}_{h}$. The proof uses a regularization in a first step to apply Brouwer's fixed-point theorem; then, solutions are shown to satisfy (3.3)-(3.4), and discrete versions of the sphere constraint and the energy law. In the following, we use the notation for the energy given in (1.1).

Lemma 3.1. Let $\mathcal{T}_{h}$ be a quasiuniform triangulation of $\Omega \subset \mathbb{R}^{d}$, and $\mathbf{U}^{0} \in \mathbf{V}_{h}$ such that $\left|\mathbf{U}^{0}(\mathbf{z})\right|=1$ for all $\mathbf{z} \in \mathcal{N}_{h}$. For sufficiently small $\tilde{C}=\tilde{C}\left(\Omega, \mathcal{T}_{h}\right)>0$ independent of $k, h>0$ such that $k \leq \tilde{C} h^{2}$, there exists $\mathbf{U}^{n+1} \in \mathbf{V}_{h}$ which satisfies (3.3) -(3.4), $\left|\mathbf{U}^{n+1}(\mathbf{z})\right|=1$ for all $\mathbf{z} \in \mathcal{N}_{h}$, and

$$
E\left(\mathbf{U}^{N+1}\right)+k \sum_{n=0}^{N}\left\|d_{t} \mathbf{U}^{n+1}\right\|_{h}^{2}=E\left(\mathbf{U}^{0}\right) \quad(N \geq 0) .
$$

Proof. Step 1. Fix $n \geq 0$. For every $\frac{1}{8}<\varepsilon \leq \frac{1}{4}$, and all $\boldsymbol{\Phi} \in \mathbf{V}_{h}$, define the continuous mapping $\mathbf{F}_{\varepsilon}: \mathbf{V}_{h} \rightarrow \mathbf{V}_{h}$, where

$$
\begin{aligned}
\left(\mathbf{F}_{\varepsilon}(\mathbf{W}), \boldsymbol{\Phi}\right):= & \left(\frac{2}{k}\left\{\mathbf{W}-\mathbf{U}^{n}\right\}, \boldsymbol{\Phi}\right)_{h}+(\nabla \mathbf{W}, \nabla \boldsymbol{\Phi}) \\
& -\sum_{\mathbf{z} \in \mathcal{N}_{h}}\left(\frac{\left(\nabla \mathbf{W}, \mathbf{W}(\mathbf{z}) \otimes \nabla \varphi_{\mathbf{z}}\right)}{\beta_{\mathbf{z}} \max \left\{|\mathbf{W}(\mathbf{z})|^{2}, \varepsilon\right\}} \varphi_{\mathbf{z}} \mathbf{W}, \boldsymbol{\Phi}\right)_{h} .
\end{aligned}
$$

We compute

$$
\begin{aligned}
& \left(\frac{\beta_{\mathbf{z}}^{-1}\left(\nabla \mathbf{W}, \mathbf{W}(\mathbf{z}) \otimes \nabla \varphi_{\mathbf{z}}\right)}{\max \left\{|\mathbf{W}(\mathbf{z})|^{2}, \varepsilon\right\}} \varphi_{\mathbf{z}},|\mathbf{W}|^{2}\right)_{h} \\
& \quad=\frac{|\mathbf{W}(\mathbf{z})|^{2}}{\max \left\{|\mathbf{W}(\mathbf{z})|^{2}, \varepsilon\right\}}\left(\nabla \mathbf{W}, \mathbf{W}(\mathbf{z}) \otimes \nabla \varphi_{\mathbf{z}}\right) \\
& \quad \leq\left(|\nabla \mathbf{W}|,\left|\nabla\left[\mathbf{W}(\mathbf{z}) \varphi_{\mathbf{z}}\right]\right|\right)_{\operatorname{supp}\left(\nabla \varphi_{\mathbf{z}}\right)} \\
& \quad \leq C h^{-1}(|\nabla \mathbf{W}|,|\mathbf{W}(\mathbf{z})|)_{\operatorname{supp}\left(\nabla \varphi_{\mathbf{z}}\right)} .
\end{aligned}
$$


For all $\mathbf{W}=\boldsymbol{\Phi}$ such that $\|\mathbf{W}\|_{h} \geq\left\|\mathbf{U}^{n}\right\|_{h}$, and values $k \leq \tilde{C} h^{2}$ for some existing $0<\tilde{C} \equiv \tilde{C}(\Omega)$, on using Young's inequality, and the fact that the number of nodes $\mathbf{y} \in \mathcal{N}_{h}$ such that $\left(\nabla \varphi_{\mathbf{y}}, \nabla \varphi_{\mathbf{z}}\right) \neq 0$ is bounded independently of $h>0$,

$$
\begin{aligned}
\left(\mathbf{F}_{\varepsilon}(\mathbf{W}), \mathbf{W}\right) & \geq \frac{2}{k}\left(\|\mathbf{W}\|_{h}^{2}-\left(\mathbf{U}^{n}, \mathbf{W}\right)_{h}\right)+\|\nabla \mathbf{W}\|^{2}-C h^{-1}\|\nabla \mathbf{W}\|\|\mathbf{W}\|_{h} \\
& \geq \frac{2}{k}\|\mathbf{W}\|_{h}\left(\left(1-\frac{C k}{h^{2}}\right)\|\mathbf{W}\|_{h}-\left\|\mathbf{U}^{n}\right\|_{h}\right)+\frac{1}{2}\|\nabla \mathbf{W}\|^{2} \geq 0,
\end{aligned}
$$

and a corollary to Brouwer's fixed-point theorem [21, p. 37] then implies existence of $\mathbf{U}^{n+1 / 2} \in \mathbf{V}_{h}$, such that $\mathbf{F}_{\varepsilon}\left(\mathbf{U}^{n+1 / 2}\right)=0$.

Step 2. We proceed by induction to show that $\mathbf{U}^{n+1 / 2} \in \mathbf{V}_{h}$ solves $\mathbf{F}_{0}\left(\mathbf{U}^{n+1 / 2}\right)=$ $\mathbf{0}$, provided $k \leq \tilde{C} h^{2}$. Let $n \geq 1$. For all $0 \leq \ell \leq n$, suppose that $\mathbf{U}^{\ell} \in \mathbf{V}_{h}$ satisfies

$$
\left|\mathbf{U}^{\ell}(\mathbf{z})\right|=1 \quad \forall \mathbf{z} \in \mathcal{N}_{h}, \quad E\left(\mathbf{U}^{\ell}\right)+\frac{k}{2} \sum_{j=0}^{\ell-1}\left\|d_{t} \mathbf{U}^{j+1}\right\|_{h}^{2}=E\left(\mathbf{U}^{0}\right) .
$$

In order to validate $\mathbf{F}_{0}\left(\mathbf{U}^{n+1 / 2}\right)=\mathbf{0}$, it suffices to show for all $\mathbf{z} \in \mathcal{N}_{h}$ (by the triangle inequality)

$$
\left|\mathbf{U}^{n+1 / 2}(\mathbf{z})\right| \geq 1-\frac{k}{2}\left|d_{t} \mathbf{U}^{n+1}(\mathbf{z})\right| \stackrel{!}{>} \frac{1}{2}
$$

By (3.6), the iterate $\mathbf{U}^{n+1}=2 \mathbf{U}^{n+1 / 2}-\mathbf{U}^{n}$ satisfies for all $\boldsymbol{\Phi} \in \mathbf{V}_{h}$,

$$
\begin{aligned}
& \left(d_{t} \mathbf{U}^{n+1}, \boldsymbol{\Phi}\right)_{h}+\left(\nabla \mathbf{U}^{n+1 / 2}, \nabla \boldsymbol{\Phi}\right) \\
& \quad=\sum_{\mathbf{z} \in \mathcal{N}_{h}}\left(\frac{\left(\nabla \mathbf{U}^{n+1 / 2}, \mathbf{U}^{n+1 / 2}(\mathbf{z}) \otimes \nabla \varphi_{\mathbf{z}}\right)}{\beta_{\mathbf{z}} \max \left\{\left|\mathbf{U}^{n+1 / 2}(\mathbf{z})\right|^{2}, \varepsilon\right\}} \varphi_{\mathbf{z}} \mathbf{U}^{n+1 / 2}, \boldsymbol{\Phi}\right)_{h} .
\end{aligned}
$$

We put $\boldsymbol{\Phi}=d_{t} \mathbf{U}^{n+1}\left(\mathbf{z}^{*}\right) \varphi_{\mathbf{z}}$ for $\mathbf{z}^{*}=\operatorname{argmax}_{\mathbf{y} \in \mathcal{N}_{h}}\left|d_{t} \mathbf{U}^{n+1}(\mathbf{y})\right|$, and use properties of reduced integration, an inverse estimate, and $\left\|\nabla \varphi_{\mathbf{z}^{*}}\right\|_{L^{1}} \leq \beta_{\mathbf{z}^{*}} h^{-1}$ to obtain

$$
\begin{aligned}
\beta_{\mathbf{z}^{*}}\left|d_{t} \mathbf{U}^{n+1}\left(\mathbf{z}^{*}\right)\right|^{2} \leq & \left|\left(\nabla \mathbf{U}^{n+1 / 2}, d_{t} \mathbf{U}^{n+1}\left(\mathbf{z}^{*}\right) \otimes \nabla \varphi_{\mathbf{z}^{*}}\right)\right| \\
& +C\left\|\nabla \mathbf{U}^{n+1 / 2}\right\|_{L^{\infty}}\left\|\nabla \varphi_{\mathbf{z}^{*}}\right\|_{L^{1}}\left|d_{t} \mathbf{U}^{n+1}\left(\mathbf{z}^{*}\right)\right| \\
\leq & C\left|\frac{k}{2}\left\|\nabla d_{t} \mathbf{U}^{n+1}\right\|_{L^{\infty}}+\left\|\nabla \mathbf{U}^{n}\right\|_{L^{\infty}}\right|\left|d_{t} \mathbf{U}^{n+1}\left(\mathbf{z}^{*}\right)\right|\left\|\nabla \varphi_{\mathbf{z}^{*}}\right\|_{L^{1}} \\
\leq & C \beta_{\mathbf{z}^{*}} h^{-2}\left[\frac{k}{2}\left|d_{t} \mathbf{U}^{n+1}\left(\mathbf{z}^{*}\right)\right|+\left\|\mathbf{U}^{n}\right\|_{L^{\infty}}\right]\left|d_{t} \mathbf{U}^{n+1}\left(\mathbf{z}^{*}\right)\right| .
\end{aligned}
$$

By assumption (3.8) for all $0 \leq \ell \leq n$, we then arrive at

$$
\left(1-\frac{C k}{h^{2}}\right)\left|d_{t} \mathbf{U}^{n+1}\left(\mathbf{z}^{*}\right)\right|^{2} \leq \tilde{C} h^{-2},
$$

for some $\tilde{C} \equiv \tilde{C}(\Omega)>0$. Hence, assertion (3.9) is valid for values $k \leq \tilde{C} h^{2}$, and consequently $\mathbf{F}_{0}\left(\mathbf{U}^{n+1 / 2}\right)=\mathbf{0}$. Therefore, upon testing (3.3) with $\boldsymbol{\Phi}=\mathbf{U}^{n+1 / 2}(\mathbf{z}) \varphi_{\mathbf{z}}$ and using the definition of $\lambda^{n+1}$, we verify that $\left|\mathbf{U}^{n+1}(\mathbf{z})\right|=1$ for all $\mathbf{z} \in \mathcal{N}_{h}$. Moreover, the energy bound (3.8) holds for all $0 \leq \ell \leq n+1$. This finishes the inductive argument. The asserted energy law follows as (1.10).

Convergence behavior of iterates $\left\{\mathbf{U}^{n}\right\}$ of Algorithm A towards weak solutions of (1.2)-(1.4) for $(k, h) \rightarrow 0$ is verified below. In the sequel, we define $\mathbf{U}_{k, h}: \Omega_{T} \rightarrow$ $\mathbb{R}^{m}$, where for all $(t, \mathbf{x}) \in\left[t_{n}, t_{n+1}\right] \times \Omega$,

$$
\mathbf{U}_{k, h}(t, \mathbf{x}):=\frac{t-t_{n}}{k} \mathbf{U}^{n+1}(\mathbf{x})+\frac{t_{n+1}-t}{k} \mathbf{U}^{n}(\mathbf{x})
$$


and $\mathbf{U}_{k, h}^{+}(t, \mathbf{x}):=\mathbf{U}^{n+1}(\mathbf{x})$, respectively, $\overline{\mathbf{U}}_{k, h}(t, \mathbf{x}):=\mathbf{U}^{n+1 / 2}(\mathbf{x})$ for all $(t, \mathbf{x}) \in$ $\left[t_{n}, t_{n+1}\right) \times \Omega$.

Theorem 3.1. Let the assumptions of Lemma 3.1 be valid, $E\left(\mathbf{u}_{0}\right)<\infty$, and $\mathbf{U}^{0} \rightarrow \mathbf{u}_{0} \in W^{1,2}\left(\Omega, \mathbb{S}^{m-1}\right)$ for $h \rightarrow 0$. There exists a subsequence of $\left\{\mathbf{U}_{k, h}\right\}$ which for $(k, h) \rightarrow 0$ converges weakly in $W^{1,2}\left(\Omega_{T}, \mathbb{R}^{m}\right)$ to a weak solution of (1.2)-(1.4).

Proof. Step 1. The bounds of Lemma 3.1 yield the existence of convergent subsequences $\left\{\mathbf{U}_{k, h}\right\}$, and $\mathbf{u} \in W^{1,2}\left(\Omega_{T}, \mathbb{R}^{m}\right)$ such that for $k \leq \tilde{C} h^{2}$, and $h \rightarrow 0$,

$$
\begin{aligned}
& \mathbf{U}_{k, h}, \mathbf{U}_{k, h}^{+}, \overline{\mathbf{U}}_{k, h} \quad \stackrel{*}{\rightarrow} \quad \mathbf{u} \quad \text { in } L^{\infty}\left(0, T ; W^{1,2}\left(\Omega, \mathbb{R}^{m}\right)\right), \\
& \mathbf{U}_{k, h}, \mathbf{U}_{k, h}^{+}, \overline{\mathbf{U}}_{k, h} \rightarrow \mathbf{u} \quad \text { in } L^{2}\left(\Omega_{T}, \mathbb{R}^{m}\right), \\
& \partial_{t} \mathbf{U}_{k, h} \quad \rightarrow \quad \mathbf{u}_{t} \quad \text { in } L^{2}\left(\Omega_{T}, \mathbb{R}^{m}\right) .
\end{aligned}
$$

Here, we use (3.5) again to conclude that sequences $\left\{\mathbf{U}_{k, h}\right\},\left\{\mathbf{U}_{k, h}^{+}\right\}$, and $\left\{\overline{\mathbf{U}}_{k, h}\right\}$ converge to the same limit as $k, h \rightarrow 0$, since

$$
\begin{aligned}
\left\|\mathbf{U}_{k, h}-\mathbf{U}_{k, h}^{+}\right\|_{L^{2}\left(0, T ; L^{2}\right)}^{2} & \leq C \sum_{n=1}^{N}\left\|\mathbf{U}^{n}-\mathbf{U}^{n-1}\right\|_{h}^{2} \int_{t_{n-1}}^{t_{n}}\left(\frac{s-t_{n}}{k}\right)^{2} \mathrm{~d} s \\
& =C \frac{k^{3}}{3} \sum_{n=1}^{N}\left\|d_{t} \mathbf{U}^{n}\right\|^{2} \rightarrow 0 .
\end{aligned}
$$

Since $\left|\mathbf{U}_{k, h}^{+}\right|=1$ for all $\mathbf{z} \in \mathcal{N}_{h}$ and all $t \in[0, T]$, there holds $\mathcal{I}_{h}\left[\left|\mathbf{U}_{k, h}^{+}\right|^{2}\right]=1$ for all $(t, \mathbf{x}) \in[0, T] \times \bar{\Omega}$, and for all $K \in \mathcal{T}_{h}$,

$$
\begin{aligned}
\left\|\left|\mathbf{U}_{k, h}^{+}\right|^{2}-1\right\|_{L^{2}(K)} & \leq C h\left\|\nabla\left[\left|\mathbf{U}_{k, h}^{+}\right|^{2}-1\right]\right\|_{L^{2}(K)} \\
& \leq C h\left\|\left(\mathbf{U}_{k, h}^{+}\right)^{T} \nabla \mathbf{U}_{k, h}^{+}\right\|_{L^{2}(K)} \leq C h\left\|\nabla \mathbf{U}_{k, h}^{+}\right\|_{L^{2}(K)} .
\end{aligned}
$$

As a consequence, $\left|\mathbf{U}_{k, h}^{+}\right| \rightarrow 1$ almost everywhere in $\Omega_{T}$, and hence $|\mathbf{u}|=1$ almost everywhere.

We use weak lower semicontinuity of norms and $\mathbf{U}^{0} \rightarrow \mathbf{u}_{0}$ in $W^{1,2}\left(\Omega, \mathbb{R}^{m}\right)$ to conclude from (3.5) that $\mathbf{u} \in W^{1,2}\left(\Omega, \mathbb{S}^{m-1}\right)$ satisfies (3.1). Since the trace operator is bounded and linear, it is weakly continuous as an operator from $W^{1,2}\left(\Omega_{T}\right)$ into $L^{2}(\Omega)$, and we deduce $\mathbf{u}(0, \cdot)=\mathbf{u}_{0}$ in the sense of traces.

Step 2. It remains to verify property (3.2) for $\mathbf{u}$. For this purpose, we rewrite (3.3) as

$$
\begin{aligned}
& \left(\partial_{t} \mathbf{U}_{k, h}(t, \cdot), \boldsymbol{\Phi}\right)_{h}+\left(\nabla \overline{\mathbf{U}}_{k, h}(t, \cdot), \nabla \boldsymbol{\Phi}\right) \\
& \quad=\sum_{\mathbf{z} \in \mathcal{N}_{h}}\left(\frac{\left(\nabla \overline{\mathbf{U}}_{k, h}(t, \cdot), \overline{\mathbf{U}}_{k, h}(t, \mathbf{z}) \otimes \nabla \varphi_{\mathbf{z}}\right)}{\beta_{\mathbf{z}}\left|\overline{\mathbf{U}}_{k, h}(\mathbf{z})\right|^{2}} \varphi_{\mathbf{z}} \overline{\mathbf{U}}_{k, h}(t, \cdot), \boldsymbol{\Phi}\right)_{h}
\end{aligned}
$$

for $\boldsymbol{\Phi} \in \mathbf{V}_{h}$, and all $t \in(0, T)$. Let $\boldsymbol{\Psi} \in C^{\infty}\left(\overline{\Omega_{T}}, \mathbb{R}^{m}\right)$; thanks to $\langle\mathbf{a} \wedge \mathbf{b}, \mathbf{a}\rangle=0$, the choice $\boldsymbol{\Phi}=\boldsymbol{I}_{h}\left[\overline{\mathbf{U}}_{k, h}(t, \cdot) \wedge \boldsymbol{\Psi}(t, \cdot)\right]$ then leads to

$$
\left(\partial_{t} \mathbf{U}_{k, h}(t, \cdot), \overline{\mathbf{U}}_{k, h}(t, \cdot) \wedge \boldsymbol{\Psi}(t, \cdot)\right)_{h}+\left(\nabla \overline{\mathbf{U}}_{k, h}(t, \cdot), \nabla \boldsymbol{I}_{h}\left[\overline{\mathbf{U}}_{k, h}(t, \cdot) \wedge \boldsymbol{\Psi}(t, \cdot)\right]\right)=0
$$


We compute

$$
\begin{aligned}
\left(\partial_{t} \mathbf{U}_{k, h}, \overline{\mathbf{U}}_{k, h} \wedge \boldsymbol{\Psi}\right)_{h}-\left(\partial_{t} \mathbf{u}, \mathbf{u} \wedge \boldsymbol{\Psi}\right) \\
=\left(\partial_{t} \mathbf{U}_{k, h}, \mathcal{I}_{h}\left[\overline{\mathbf{U}}_{k, h} \wedge \boldsymbol{\Psi}\right]\right)_{h}-\left(\partial_{t} \mathbf{U}_{k, h}, \mathcal{I}_{h}\left[\overline{\mathbf{U}}_{k, h} \wedge \boldsymbol{\Psi}\right]\right) \\
\quad+\left(\partial_{t} \mathbf{U}_{k, h}, \bar{I}_{h}\left[\overline{\mathbf{U}}_{k, h} \wedge \boldsymbol{\Psi}\right]-\overline{\mathbf{U}}_{k, h} \wedge \boldsymbol{\Psi}\right) \\
\quad+\left(\partial_{t} \mathbf{U}_{k, h},\left[\overline{\mathbf{U}}_{k, h}-\mathbf{u}\right] \wedge \boldsymbol{\Psi}\right)+\left(\partial_{t}\left[\overline{\mathbf{U}}_{k, h}-\mathbf{u}\right], \mathbf{u} \wedge \boldsymbol{\Psi}\right)
\end{aligned}
$$

The properties of $(\cdot, \cdot)_{h}, W^{1,2}(\Omega)$-stability of $\mathcal{I}_{h}$ for elementwise smooth functions, and $\left\|\overline{\mathbf{U}}_{k, h}\right\|_{L^{\infty}} \leq 1$ yield

$$
\begin{aligned}
& \left|\left(\partial_{t} \mathbf{U}_{k, h}, \mathcal{I}_{h}\left[\overline{\mathbf{U}}_{k, h} \wedge \boldsymbol{\Psi}\right]\right)_{h}-\left(\partial_{t} \mathbf{U}_{k, h}, \boldsymbol{I}_{h}\left[\overline{\mathbf{U}}_{k, h} \wedge \boldsymbol{\Psi}\right]\right)\right| \\
& \quad \leq C h\left\|\partial_{t} \mathbf{U}_{k, h}\right\|\left\|\nabla \boldsymbol{I}_{h}\left[\overline{\mathbf{U}}_{k, h} \wedge \boldsymbol{\Psi}\right]\right\| \leq C h\left\|\partial_{t} \mathbf{U}_{k, h}\right\|\left(\left\|\nabla \overline{\mathbf{U}}_{k, h}\right\|+1\right)\|\boldsymbol{\Psi}\|_{W^{1, \infty}} .
\end{aligned}
$$

Similarly,

$$
\left|\left(\partial_{t} \mathbf{U}_{k, h}, \mathcal{I}_{h}\left[\overline{\mathbf{U}}_{k, h} \wedge \boldsymbol{\Psi}\right]-\overline{\mathbf{U}}_{k, h} \wedge \boldsymbol{\Psi}\right)\right| \leq C h\left\|\partial_{t} \mathbf{U}_{k, h}\right\|\left(\left\|\nabla \overline{\mathbf{U}}_{k, h}\right\|+1\right)\|\Psi\|_{W^{1, \infty}} .
$$

Convergence towards zero $(h \rightarrow 0)$ of the last two terms in (3.13) follows from $\overline{\mathbf{U}}_{k, h} \rightarrow \mathbf{u}$ in $L^{2}\left(\Omega_{T}, \mathbb{R}^{m}\right)$, and $\partial_{t} \mathbf{U}_{k, h} \rightarrow \partial_{t} \mathbf{u}$ in $L^{2}\left(\Omega_{T}, \mathbb{R}^{m}\right)$, and (3.1), (3.5). Summing up, we find for $k \leq \tilde{C} h^{2}$,

$$
\lim _{k, h \rightarrow 0} \int_{0}^{T}\left(\partial_{t} \mathbf{U}_{k, h}, \overline{\mathbf{U}}_{k, h} \wedge \boldsymbol{\Psi}\right)_{h} \mathrm{~d} t=\int_{0}^{T}\left(\partial_{t} \mathbf{u}, \mathbf{u} \wedge \boldsymbol{\Psi}\right) \mathrm{d} t \quad \forall \boldsymbol{\Psi} \in C^{\infty}\left(\overline{\Omega_{T}}, \mathbb{R}^{m}\right) .
$$

Next, we wish to verify for the second term in (3.12) that in case $k \leq \tilde{C} h^{2}$,

$$
\begin{aligned}
\lim _{k, h \rightarrow 0} & \int_{0}^{T}\left(\nabla \overline{\mathbf{U}}_{k, h}, \nabla \mathcal{I}_{h}\left[\overline{\mathbf{U}}_{k, h} \wedge \boldsymbol{\Psi}\right]\right) \mathrm{d} t \\
& =\int_{0}^{T}(\nabla \mathbf{u}, \nabla[\mathbf{u} \wedge \boldsymbol{\Psi}]) \mathrm{d} t \quad \forall \boldsymbol{\Psi} \in C^{\infty}\left(\overline{\Omega_{T}}, \mathbb{R}^{m}\right) .
\end{aligned}
$$

Therefore, on using the identities $\left\langle\nabla \overline{\mathbf{U}}_{k, h}, \nabla\left\{\overline{\mathbf{U}}_{k, h} \wedge \boldsymbol{\Psi}\right\}\right\rangle=\left\langle\nabla \overline{\mathbf{U}}_{k, h}, \overline{\mathbf{U}}_{k, h} \wedge \nabla \boldsymbol{\Psi}\right\rangle$, and $\langle\nabla \mathbf{u}, \nabla\{\mathbf{u} \wedge \boldsymbol{\Psi}\}\rangle=\langle\nabla \mathbf{u}, \mathbf{u} \wedge \nabla \boldsymbol{\Psi}\rangle$ almost everywhere,

$$
\begin{aligned}
\int_{0}^{T}( & \left.\nabla \overline{\mathbf{U}}_{k, h}, \nabla \boldsymbol{I}_{h}\left[\overline{\mathbf{U}}_{k, h} \wedge \boldsymbol{\Psi}\right]\right)-(\nabla \mathbf{u}, \nabla[\mathbf{u} \wedge \boldsymbol{\Psi}]) \mathrm{d} t \\
= & \int_{0}^{T}\left(\nabla \overline{\mathbf{U}}_{k, h}, \nabla\left\{\mathcal{I}_{h}\left[\overline{\mathbf{U}}_{k, h} \wedge \boldsymbol{\Psi}\right]-\overline{\mathbf{U}}_{k, h} \wedge \boldsymbol{\Psi}\right\}\right) \mathrm{d} t \\
& +\int_{0}^{T}\left(\nabla \overline{\mathbf{U}}_{k, h},\left[\overline{\mathbf{U}}_{k, h}-\mathbf{u}\right] \wedge \nabla \boldsymbol{\Psi}\right) \mathrm{d} t \\
& +\int_{0}^{T}\left(\nabla\left[\overline{\mathbf{U}}_{k, h}-\mathbf{u}\right], \mathbf{u} \wedge \nabla \boldsymbol{\Psi}\right) \mathrm{d} t=: I+I I+I I I .
\end{aligned}
$$

We compute $I \leq C h\left\|\nabla \overline{\mathbf{U}}_{k, h}\right\|\left(\left\|\nabla \overline{\mathbf{U}}_{k, h}\right\|+1\right)\|\Psi\|_{W^{2, \infty}}$, by an interpolation estimate, using $\left.D^{2} \overline{\mathbf{U}}_{k, h}\right|_{K}=0$ for all $K \in \mathcal{T}_{h}$. For the terms $I I$ and $I I I$, we use $\overline{\mathbf{U}}_{k, h} \rightarrow \mathbf{u}$ in $L^{2}\left(\Omega_{T}, \mathbb{R}^{m}\right)$, and $\nabla \mathbf{U}_{k, h} \rightarrow \nabla \mathbf{u}$ in $L^{2}\left(\Omega_{T}, \mathbb{R}^{m}\right)$, respectively, to conclude that $I I, I I I \rightarrow 0$, for $k \leq \tilde{C} h^{2}$, and $(k, h) \rightarrow 0$. Therefore, the limit $\mathbf{u}: \Omega_{T} \rightarrow \mathbb{R}^{m}$ satisfies (3.2). 


\section{WAVE MAP to THE SPHERE}

We recall the notion of weak solutions to (1.5)-(1.7) from [20] in the following definition. Below, let $E(\mathbf{v}, \mathbf{w}):=\frac{1}{2}\left[\|\mathbf{v}\|^{2}+\|\nabla \mathbf{w}\|^{2}\right]$.

Definition 4.1. Given $T>0$ and $\left(\mathbf{u}_{0}, \mathbf{v}_{0}\right) \in W^{1,2}\left(\Omega, \mathbb{S}^{m-1}\right) \times L^{2}\left(\Omega, \mathbb{R}^{m}\right)$ with $\left\langle\mathbf{u}_{0}, \mathbf{v}_{0}\right\rangle=0$ a.e. in $\Omega$, we call $\mathbf{u}: \Omega_{T} \rightarrow \mathbb{R}^{m}$ a weak solution of (1.5)- (1.7) if (i) $D \mathbf{u} \in L^{2}\left(\Omega_{T}, \mathbb{R}^{m}\right)$, (ii) $|\mathbf{u}|=1$ almost everywhere in $\Omega_{T}$,

$$
\begin{aligned}
& -\int_{0}^{T}\left(\mathbf{u}_{t} \wedge \mathbf{u}, \boldsymbol{\phi}_{t}\right) \mathrm{d} t+\int_{0}^{T}(\nabla \mathbf{u} \wedge \mathbf{u}, \nabla \boldsymbol{\phi}) \mathrm{d} t=\left(\mathbf{v}_{0} \wedge \mathbf{u}_{0}, \boldsymbol{\phi}(0, \cdot)\right) \\
& \forall \boldsymbol{\phi} \in C_{0}^{\infty}\left([0, T) ; W^{1,2}\left(\Omega_{T}, \mathbb{R}^{m}\right)\right), \\
& E\left(\mathbf{u}_{t}(t, \cdot), \mathbf{u}(t, \cdot)\right) \leq E\left(\mathbf{v}_{0}, \mathbf{u}_{0}\right) \quad \text { for a.e. } t \geq 0, \\
& \mathbf{u}(t, \cdot) \rightarrow \mathbf{u}_{0} \quad \text { in } W^{1,2}\left(\Omega ; \mathbb{R}^{m}\right), \quad \mathbf{u}_{t}(t, \cdot) \rightarrow \mathbf{v}_{0} \quad \text { in } L^{2}\left(\Omega, \mathbb{R}^{m}\right) \quad(t \rightarrow 0) .
\end{aligned}
$$

We numerically approximate weak solutions of (1.5)-1.7). Next, we give an explicit formula for $\lambda^{n+1}$ in Algorithm B to study well-posedness, which is again obtained by choosing $\boldsymbol{\Phi}=\mathbf{U}^{n+1 / 2}(\mathbf{z}) \varphi_{\mathbf{z}}$.

Algorithm B. Given $\mathbf{U}^{n}, \mathbf{U}^{n-1} \in \mathbf{V}_{h}$, find $\left(\mathbf{U}^{n+1}, \lambda^{n+1}\right) \in \mathbf{V}_{h} \times V_{h}$, such that for all $\mathbf{\Phi} \in \mathbf{V}_{h}$ and all $\mathbf{z} \in \mathcal{N}_{h}$, there holds

$$
\begin{gathered}
\left(d_{t}^{2} \mathbf{U}^{n+1}, \boldsymbol{\Phi}\right)_{h}+\left(\nabla \mathbf{U}^{n+1 / 2}, \nabla \boldsymbol{\Phi}\right)=\left(\lambda^{n+1} \mathbf{U}^{n+1 / 2}, \boldsymbol{\Phi}\right)_{h}, \\
\lambda^{n+1}(\mathbf{z})= \begin{cases}0 \quad \text { for } \mathbf{U}^{n+1 / 2}(\mathbf{z})=0 \\
\frac{-\frac{1}{2}\left[\left|d_{t} \mathbf{U}^{n}(\mathbf{z})\right|^{2}+\left\langle d_{t} \mathbf{U}^{n}(\mathbf{z}), d_{t} \mathbf{U}^{n+1}(\mathbf{z})\right\rangle\right]+\left(\nabla \mathbf{U}^{n+1 / 2}(\mathbf{z}), \mathbf{U}^{n+1 / 2}(\mathbf{z}) \otimes \nabla \varphi_{\mathbf{z}}\right)}{\beta_{\mathbf{z}}\left|\mathbf{U}^{n+1 / 2}(\mathbf{z})\right|^{2}} & \text { else. }\end{cases}
\end{gathered}
$$

We set $\mathbf{U}^{-1}=\mathbf{U}^{0}-k \mathbf{V}^{0}$ with the given initial velocity $\mathbf{V}^{0}$, so that $\mathbf{V}^{0}=d_{t} \mathbf{U}^{0}$, and use the above algorithm for $n \geq 0$. We let

$$
\mathbf{V}^{n}=d_{t} \mathbf{U}^{n} \quad \text { for } n \geq 1 \text {. }
$$

Below, the discrete energy is denoted as

$$
E_{h}(\mathbf{V}, \mathbf{U})=\frac{1}{2}\left(\|\mathbf{V}\|_{h}^{2}+\|\nabla \mathbf{U}\|^{2}\right)
$$

In the following, the time step restriction $k=\mathcal{O}\left(h^{\max \{d / 2,1\}}\right)$ and quasiuniform meshes $\mathcal{T}_{h}$ are sufficient to verify solvability of Algorithm B. The proof uses a regularization in a first step to apply Brouwer's theorem; then, solutions are shown to satisfy discrete versions of the sphere constraint and the energy law in the case of a mesh constraint, and converge to weak solutions of (1.5)-(1.7).

Lemma 4.1. Let $\mathcal{T}_{h}$ be a quasiuniform triangulation of $\Omega \subset \mathbb{R}^{d}$, and $\left(\mathbf{U}^{0}, \mathbf{V}^{0}\right) \in$ $\mathbf{V}_{h} \times \mathbf{V}_{h}$ with $\left|\mathbf{U}^{0}(\mathbf{z})\right|=1$ and $\left\langle\mathbf{U}^{0}(\mathbf{z}), \mathbf{V}^{0}(\mathbf{z})\right\rangle=0$ for all $\mathbf{z} \in \mathcal{N}_{h}$. For $n \geq 1$, for sufficiently small $\tilde{C}=\tilde{C}\left(\Omega, \mathcal{T}_{h}\right)>0$ independent of $k, h>0$ such that $k \leq$ $\tilde{C} h^{\max \{d / 2,1\}}$, there exists $\mathbf{U}^{n+1} \in \mathbf{V}_{h}$, which satisfies Algorithm $B,\left|\mathbf{U}^{n+1}(\mathbf{z})\right|=1$ for all $\mathbf{z} \in \mathcal{N}_{h}$, and

$$
E_{h}\left(\mathbf{V}^{N+1}, \mathbf{U}^{N+1}\right)+\frac{k^{2}}{2} \sum_{n=0}^{N}\left\|d_{t} \mathbf{V}^{n+1}\right\|_{h}^{2}=E_{h}\left(\mathbf{V}^{0}, \mathbf{U}^{0}\right) \quad(N \geq 0) .
$$

A verification of this lemma follows the steps of the proof of Lemma 3.1 adapted to the present case. 
Proof. The discrete energy law (4.1) follows from the first equation in Algorithm $\mathrm{B}$, on choosing $\boldsymbol{\Phi}=d_{t} \mathbf{U}^{n+1}$.

Step 1. Fix $n \geq 0$. For every $\frac{1}{8}<\varepsilon \leq \frac{1}{4}$, and all $\boldsymbol{\Phi} \in \mathbf{V}_{h}$, define the continuous function $\mathbf{F}_{\varepsilon}: \mathbf{V}_{h} \rightarrow \mathbf{V}_{h}$, where

$$
\begin{aligned}
\left(\mathbf{F}_{\varepsilon}(\mathbf{W}), \boldsymbol{\Phi}\right):= & \frac{2}{k^{2}}\left(\mathbf{W}+\mathbf{U}^{n-1 / 2}-2 \mathbf{U}^{n}, \boldsymbol{\Phi}\right)_{h}+(\nabla \mathbf{W}, \nabla \boldsymbol{\Phi}) \\
& -\sum_{\mathbf{z} \in \mathcal{N}_{h}}\left[I_{\mathbf{z}}^{\varepsilon}(\mathbf{W} ; \boldsymbol{\Phi})+I I_{\mathbf{z}}^{\varepsilon}(\mathbf{W} ; \boldsymbol{\Phi})\right],
\end{aligned}
$$

for

$$
\begin{aligned}
I_{\mathbf{z}}^{\varepsilon}(\mathbf{W} ; \boldsymbol{\Phi}) & :=\left(\frac{-\frac{1}{2}\left[\left|d_{t} \mathbf{U}^{n}(\mathbf{z})\right|^{2}+\frac{2}{k}\left\langle d_{t} \mathbf{U}^{n}(\mathbf{z}), \mathbf{W}(\mathbf{z})-\mathbf{U}^{n}(\mathbf{z})\right\rangle\right]}{\beta_{\mathbf{z}} \max \left\{|\mathbf{W}|^{2}, \varepsilon\right\}} \varphi_{\mathbf{z}} \mathbf{W}, \boldsymbol{\Phi}\right)_{h} \\
I I_{\mathbf{z}}^{\varepsilon}(\mathbf{W} ; \boldsymbol{\Phi}) & :=\left(\frac{\left(\nabla \mathbf{W}, \mathbf{W}(\mathbf{z}) \otimes \nabla \varphi_{\mathbf{z}}\right)}{\beta_{\mathbf{z}} \max \left\{|\mathbf{W}|^{2}, \varepsilon\right\}} \varphi_{\mathbf{z}} \mathbf{W}, \boldsymbol{\Phi}\right)_{h} .
\end{aligned}
$$

We compute

$$
\begin{aligned}
\sum_{\mathbf{z} \in \mathcal{N}_{h}} I_{\mathbf{z}}^{\varepsilon}\left(\mathbf{W} ; \mathbf{W}(\mathbf{z}) \varphi_{\mathbf{z}}\right) & \leq \frac{|\mathbf{W}(\mathbf{z})|^{2}}{\max \left\{|\mathbf{W}(\mathbf{z})|^{2}, \varepsilon\right\}}\left[C\left\|d_{t} \mathbf{U}^{n}\right\|_{h}^{2}+\frac{1}{4 k^{2}}\left\|\mathbf{W}-\mathbf{U}^{n}\right\|_{h}^{2}\right] \\
& \leq C\left\|d_{t} \mathbf{U}^{n}\right\|_{h}^{2}+\frac{1}{4 k^{2}}\left\|\mathbf{W}-\mathbf{U}^{n}\right\|_{h}^{2},
\end{aligned}
$$

and

$$
\begin{aligned}
I I_{\mathbf{z}}^{\varepsilon}\left(\mathbf{W} ; \mathbf{W}(\mathbf{z}) \varphi_{\mathbf{z}}\right) & =\frac{|\mathbf{W}(\mathbf{z})|^{2}}{\max \left\{|\mathbf{W}(\mathbf{z})|^{2}, \varepsilon\right\}}\left(\nabla \mathbf{W}, \mathbf{W}(\mathbf{z}) \otimes \nabla \varphi_{\mathbf{z}}\right) \\
& \leq\left(|\nabla \mathbf{W}|,\left|\nabla\left[\mathbf{W}(\mathbf{z}) \varphi_{\mathbf{z}}\right]\right|\right)_{\operatorname{supp}\left(\nabla \varphi_{\mathbf{z}}\right)} \\
& \leq C h^{-1}(|\nabla \mathbf{W}|,|\mathbf{W}(\mathbf{z})|)_{\operatorname{supp}\left(\nabla \varphi_{\mathbf{z}}\right)} .
\end{aligned}
$$

Choose $\mathbf{W}=\boldsymbol{\Phi}$, and use Young's inequality, and the fact that the number of nodes $\mathbf{y} \in \mathcal{N}_{h}$ is such that $\left(\nabla \varphi_{\mathbf{y}}, \nabla \varphi_{\mathbf{z}}\right) \neq 0$ is bounded independent from $h>0$. Estimates (4.2), (4.3), and rearranging terms lead to

$$
\begin{aligned}
\left(\mathbf{F}_{\varepsilon}(\mathbf{W}), \mathbf{W}\right)_{h} \geq & \frac{2}{k^{2}}\left(\|\mathbf{W}\|_{h}^{2}-\left|\left(\mathbf{U}^{n-1 / 2}, \mathbf{W}\right)_{h}\right|-2\left|\left(\mathbf{U}^{n}, \mathbf{W}\right)_{h}\right|\right)+\|\nabla \mathbf{W}\|^{2} \\
& -C\left\|d_{t} \mathbf{U}^{n}\right\|_{h}^{2}-\frac{1}{4 k^{2}}\left\|\mathbf{W}-\mathbf{U}^{n}\right\|_{h}^{2}-C h^{-1}\|\nabla \mathbf{W}\|\|\mathbf{W}\|_{h} \\
\geq & \frac{1}{k^{2}}\|\mathbf{W}\|_{h}\left(\left(1-\frac{C k^{2}}{h^{2}}\right)\|\mathbf{W}\|_{h}-4\left\|\mathbf{U}^{n-1 / 2}\right\|_{h}-4\left\|\mathbf{U}^{n}\right\|_{h}\right)+\frac{1}{2}\|\nabla \mathbf{W}\|^{2} \\
& -C\left\|d_{t} \mathbf{U}^{n}\right\|^{2}-\frac{1}{4 k^{2}}\left\|\mathbf{U}^{n}\right\|_{h}^{2} .
\end{aligned}
$$

Suppose that $k \leq \tilde{C} h$, for some $\tilde{C} \equiv \tilde{C}(\Omega)>0$ sufficiently small. It is then possible to find $R_{1}>0$, such that for all $\left\{\mathbf{W} \in \mathbf{V}_{h}:\|\mathbf{W}\|_{h} \geq R_{1}\right\}$ there holds

$$
\left(\mathbf{F}_{\varepsilon}(\mathbf{W}), \mathbf{W}\right)_{h} \geq \frac{1}{2 k^{2}}\|\mathbf{W}\|_{h}^{2}-C\left\|d_{t} \mathbf{U}^{n}\right\|_{h}^{2}-\frac{1}{4 k^{2}}\left\|\mathbf{U}^{n}\right\|_{h}^{2} \geq 0 .
$$

Hence, there exist some $0<R_{1} \leq R_{2}<\infty$ such that $\left(\mathbf{F}_{\varepsilon}(\mathbf{W}), \mathbf{W}\right)_{h} \geq 0$ for all $\mathbf{W} \in \mathbf{V}_{h}$, which satisfy $\|\mathbf{W}\|_{h} \geq R_{2}$. Then, Brouwer's fixed-point theorem implies existence of $\mathbf{U}^{n+1 / 2} \in \mathbf{V}_{h}$, such that $\mathbf{F}_{\varepsilon}\left(\mathbf{U}^{n+1 / 2}\right)=\mathbf{0}$. 
Step 2. We show that $\mathbf{U}^{n+1 / 2} \in \mathbf{V}_{h}$ solves $\mathbf{F}_{0}\left(\mathbf{U}^{n+1 / 2}\right)=\mathbf{0}$. For this purpose, it suffices to show for all $\mathbf{z} \in \mathcal{N}_{h}$,

$$
\left|\mathbf{U}^{n+1 / 2}(\mathbf{z})\right| \geq 1-\frac{k}{2}\left|d_{t} \mathbf{U}^{n+1}(\mathbf{z})\right| \stackrel{!}{>} \frac{1}{2} .
$$

We proceed by induction to verify this result for $k \leq \tilde{C} h^{\max \{d / 2,1\}}$. Let $n \geq 1$ : For all $0 \leq \ell \leq n$, suppose that $\mathbf{U}^{\ell} \in \mathbf{V}_{h}$ verifies $\left|\mathbf{U}^{\ell}(\mathbf{z})\right|=1$, for all $\mathbf{z} \in \mathcal{N}_{h}$, and

$$
E_{h}\left(\mathbf{V}^{n}, \mathbf{U}^{n}\right)+\frac{k^{2}}{2} \sum_{\ell=1}^{n}\left\|d_{t} \mathbf{V}^{\ell}\right\|_{h}^{2}=E_{h}\left(\mathbf{V}^{0}, \mathbf{U}^{0}\right) .
$$

The iterate $\mathbf{U}^{n+1}:=2 \mathbf{U}^{n+1 / 2}-\mathbf{U}^{n} \in \mathbf{V}_{h}$ satisfies, for all $\boldsymbol{\Phi} \in \mathbf{V}_{h}$,

$$
\left(d_{t}^{2} \mathbf{U}^{n+1}, \boldsymbol{\Phi}\right)_{h}+\left(\nabla \mathbf{U}^{n+1 / 2}, \nabla \boldsymbol{\Phi}\right)=-\sum_{\mathbf{z} \in \mathcal{N}_{h}} I_{\mathbf{z}}^{\varepsilon}\left(\mathbf{U}^{n+1 / 2} ; \boldsymbol{\Phi}\right)+I I_{\mathbf{z}}^{\varepsilon}\left(\mathbf{U}^{n+1 / 2} ; \boldsymbol{\Phi}\right) \text {. }
$$

On putting $\boldsymbol{\Phi}=d_{t} \mathbf{U}^{n+1}$, we obtain

$$
\begin{aligned}
\frac{1}{2} d_{t}[ & \left.\left\|d_{t} \mathbf{U}^{n+1}\right\|_{h}^{2}+\left\|\nabla \mathbf{U}^{n+1}\right\|^{2}\right]+\frac{k}{2}\left\|d_{t}^{2} \mathbf{U}^{n+1}\right\|_{h}^{2} \\
& =-\sum_{\mathbf{z} \in \mathcal{N}_{h}} I_{\mathbf{z}}^{\varepsilon}\left(\mathbf{U}^{n+1 / 2} ; d_{t} \mathbf{U}^{n+1}\right)+I I_{\mathbf{z}}^{\varepsilon}\left(\mathbf{U}^{n+1 / 2} ; d_{t} \mathbf{U}^{n+1}\right) .
\end{aligned}
$$

We use $k d_{t} \mathbf{U}^{n+1}=2 \mathbf{U}^{n+1 / 2}-2 \mathbf{U}^{n}$ to control

$$
\begin{aligned}
\sum_{\mathbf{z} \in \mathcal{N}_{h}} I_{\mathbf{z}}^{\varepsilon}\left|\left(\mathbf{U}^{n+1 / 2} ; d_{t} \mathbf{U}^{n+1}\right)\right| & \leq\left(\frac{1+\left|d_{t} \mathbf{U}^{n}\right|\left\{\left|d_{t} \mathbf{U}^{n+1}\right|+\left|d_{t} \mathbf{U}^{n}\right|\right\}}{\beta_{\mathbf{z}} \max \left\{\left|\mathbf{U}^{n+1 / 2}\right|, \varepsilon\right\}},\left|d_{t} \mathbf{U}^{n+1}\right|\right)_{h} \\
& \leq \frac{C}{k}\left(1+\frac{C}{\varepsilon}\left\|\mathbf{U}^{n}\right\|_{L^{\infty}}\right)\left\|d_{t} \mathbf{U}^{n}\right\|_{h}\left(\left\|d_{t} \mathbf{U}^{n}\right\|_{h}+\left\|d_{t} \mathbf{U}^{n+1}\right\|_{h}\right) .
\end{aligned}
$$

For the second term, we conclude

$$
\sum_{\mathbf{z} \in \mathcal{N}_{h}} I I_{\mathbf{z}}^{\varepsilon}\left|\left(\mathbf{U}^{n+1 / 2} ; d_{t} \mathbf{U}^{n+1}\right)\right| \leq C h^{-1}\left\|\nabla \mathbf{U}^{n+1 / 2}\right\|_{L^{2}}\left\|d_{t} \mathbf{U}^{n+1}\right\|_{h} .
$$

Putting things together yields

$$
\begin{gathered}
\frac{1}{2 k}\left(1-\frac{1}{8}-\frac{C k}{h}\right)\left\|d_{t} \mathbf{U}^{n+1}\right\|_{h}^{2}+\frac{1}{2 k}\left(1-\frac{1}{8}-\frac{C k}{h}\right)\left\|\nabla \mathbf{U}^{n+1}\right\|_{h}^{2}+\frac{k}{2}\left\|d_{t}^{2} \mathbf{U}^{n+1}\right\|_{h}^{2} \\
\leq \frac{1}{2 k}\left(1+C\left[1+\frac{C}{\varepsilon^{2}}\left\|\mathbf{U}^{n}\right\|_{L^{\infty}}^{2}\right]\right)\left\|d_{t} \mathbf{U}^{n}\right\|_{h}^{2}+C\left(\frac{1}{k}+\frac{1}{h}\right)\left\|\nabla \mathbf{U}^{n}\right\|_{h}^{2} .
\end{gathered}
$$

By inverse estimate, induction assumption, and for values $k \leq \tilde{C} h$,

$$
\frac{k^{2}}{2}\left\|d_{t} \mathbf{U}^{n+1}\right\|_{L^{\infty}}^{2} \leq C k^{2} h^{-d}\left\|d_{t} \mathbf{U}^{n+1}\right\|_{h}^{2} \leq C k^{2} h^{-d}\left[C\left(1+\frac{1}{\varepsilon^{2}}\right)\left\|d_{t} \mathbf{U}^{n}\right\|_{h}^{2}+2\left\|\nabla \mathbf{U}^{n}\right\|^{2}\right] .
$$

Hence, assertion (4.4) holds for values $k \leq \tilde{C} h^{\max \{d / 2,1\}}$, and hence $\mathbf{F}_{0}\left(\mathbf{U}^{n+1 / 2}\right)=$ 0. This implies $\left|\mathbf{U}^{n+1}(\mathbf{z})\right|=1$ for all $\mathbf{z} \in \mathcal{N}_{h}$, as well as (4.5) for $n+1$, and hence the induction argument is complete.

Step 3. The last step deletes the first possibility in (B) , provided $k \leq \tilde{C} h^{\max \{d / 2,1\}}$. In order to compute $\lambda^{n+1}(\mathbf{z})$, for every $\mathbf{z} \in \mathcal{N}_{h}$, we put $\boldsymbol{\Phi}=\mathbf{U}^{n+1 / 2}(\mathbf{z}) \varphi_{\mathbf{z}}$. For the leading term, we find

$$
\left\langle d_{t}^{2} \mathbf{U}^{n+1}(\mathbf{z}), \mathbf{U}^{n+1 / 2}(\mathbf{z})\right\rangle=-\frac{1}{2}\left[\left|d_{t} \mathbf{U}^{n}(\mathbf{z})\right|^{2}+\left\langle d_{t} \mathbf{U}^{n}(\mathbf{z}), d_{t} \mathbf{U}^{n+1}(\mathbf{z})\right\rangle\right],
$$

thanks to $d_{t}\left|\mathbf{U}^{n+1}(\mathbf{z})\right|^{2}=0$ for all $\mathbf{z} \in \mathcal{N}_{h}$. 
Convergence behavior of iterates $\left\{\mathbf{U}^{n}\right\} \subset \mathbf{V}_{h}$ of Algorithm B towards weak solutions of (1.5)-(1.7) for $(k, h) \rightarrow 0$ is verified below. In the sequel, given $\left\{\boldsymbol{\Phi}^{n}\right\} \subset$ $\mathbf{V}_{h}$, we define $\boldsymbol{\Phi}_{k, h}: \Omega_{T} \rightarrow \mathbb{R}^{m}$, where for all $(t, \mathbf{x}) \in\left[t_{n}, t_{n+1}\right) \times \Omega$,

$$
\begin{aligned}
& \boldsymbol{\Phi}_{k, h}(t, \mathbf{x}):=\frac{t-t_{n}}{k} \boldsymbol{\Phi}^{n+1}(\mathbf{x})+\frac{t_{n+1}-t}{k} \boldsymbol{\Phi}^{n}(\mathbf{x}), \\
& \boldsymbol{\Phi}_{k, h}^{+}(t, \mathbf{x}):=\boldsymbol{\Phi}^{n+1}(\mathbf{x}), \quad \overline{\boldsymbol{\Phi}}_{k, h}(t, \mathbf{x}):=\boldsymbol{\Phi}^{n+1 / 2}(\mathbf{x}) .
\end{aligned}
$$

Subsequently, we drop subindices $k, h$ and use $\left(\mathbf{U}^{+}, \overline{\mathbf{U}}, \mathbf{U}\right)$, respectively, $\left(\lambda^{+}, \lambda\right)$, and $\left(\mathbf{V}^{+}, \mathbf{V}\right)$ to stand for $\left(\mathbf{U}_{k, h}^{+}, \overline{\mathbf{U}}_{k, h}, \mathbf{U}_{k, h}\right)$, respectively, $\left(\lambda_{k, h}^{+}, \lambda_{k, h}\right)$, and $\left(\mathbf{V}_{k, h}^{+}, \mathbf{V}_{k, h}\right)$. For $\boldsymbol{\Phi} \in C_{0}^{\infty}\left([0, T) ; \mathbf{V}_{h}\right)$, on putting $\mathbf{V}^{n+1}=d_{t} \mathbf{U}^{n+1}$, we may rewrite the first equation of Algorithm B as follows:

$$
\int_{0}^{T}\left[\left(\mathbf{V}_{t}, \boldsymbol{\Phi}\right)_{h}+(\nabla \overline{\mathbf{U}}, \nabla \boldsymbol{\Phi})-\left(\lambda^{+} \overline{\mathbf{U}}, \boldsymbol{\Phi}\right)_{h}\right] \mathrm{d} t=0 .
$$

We first derive the following reformulation of Algorithm B.

Lemma 4.2. Suppose that the assumptions of Lemma 4.1 are valid. There holds for all $\Psi \in C_{0}^{\infty}\left([0, T) ; C^{\infty}\left(\Omega, \mathbb{R}^{m}\right)\right)$,

$$
\begin{gathered}
\left|\int_{0}^{T}\left[-\left(\mathbf{U}_{t},[\mathbf{U} \wedge \boldsymbol{\Psi}]_{t}\right)_{h}+\left(\nabla \overline{\mathbf{U}}, \nabla \mathcal{I}_{h}[\overline{\mathbf{U}} \wedge \boldsymbol{\Psi}]\right)\right] \mathrm{d} t-\left(\mathbf{V}^{0}, \mathbf{U}(0, \cdot) \wedge \boldsymbol{\Psi}(0, \cdot)\right)_{h}\right| \\
\leq\left|\int_{0}^{T}\left(\mathbf{V}^{+}-\mathbf{V},[\mathbf{U} \wedge \boldsymbol{\Psi}]_{t}\right)_{h} \mathrm{~d} t\right|+C k^{1 / 2} E_{h}\left(\mathbf{V}^{0}, \mathbf{U}^{0}\right)\|\boldsymbol{\Psi}\|_{L^{\infty}\left(\Omega_{T}\right)}
\end{gathered}
$$

where $\overline{\mathbf{U}}(0, \cdot)=\frac{k}{2} \mathbf{V}^{0}+\mathbf{U}^{0}$.

Proof. Let $\boldsymbol{\Psi} \in C_{0}^{\infty}\left([0, T) ; C^{\infty}\left(\Omega, \mathbb{R}^{m}\right)\right)$, and take $\boldsymbol{\Phi}=\boldsymbol{I}_{h}[\overline{\mathbf{U}} \wedge \boldsymbol{\Psi}]$ in (4.8). We then restate the integrand of the first term in (4.8) as follows:

$$
\left(\mathbf{V}_{t}, \overline{\mathbf{U}} \wedge \psi\right)_{h}=\left(\mathbf{V}_{t}, \mathbf{U} \wedge \psi\right)_{h}+\left(\mathbf{V}_{t},[\overline{\mathbf{U}}-\mathbf{U}] \wedge \psi\right)_{h}
$$

We use the following identity in $\Omega$,

$$
\overline{\mathbf{U}}(t, \cdot)=\mathbf{U}(t, \cdot)+\left[\frac{1}{2}\left(t_{n+1}+t_{n}\right)-t\right] \mathbf{V}^{+}(t, \cdot) \quad \forall t \in\left[t_{n}, t_{n+1}\right),
$$

the estimate $\left(\mathbf{V}_{t},[\overline{\mathbf{U}}-\mathbf{U}] \wedge \boldsymbol{\Psi}\right)_{h} \leq \frac{k}{2}\left\|\mathbf{V}_{t}\right\|_{h}\left\|\mathbf{V}^{+}\right\|_{h}\|\Psi\|_{L^{\infty}}$, and the energy identity (4.1) to bound the last term in (4.10) as follows:

$$
\begin{aligned}
\int_{0}^{T}\left(\mathbf{V}_{t},[\overline{\mathbf{U}}-\mathbf{U}] \wedge \boldsymbol{\Psi}\right)_{h} \mathrm{~d} t & \leq C k^{1 / 2}\left(k \int_{0}^{T}\left\|\mathbf{V}_{t}(t, \cdot)\right\|^{2} \mathrm{~d} t\right)^{1 / 2}\|\boldsymbol{\Psi}\|_{L^{\infty}\left(\Omega_{T}\right)} \\
& \leq C \sqrt{k} E_{h}\left(\mathbf{V}^{0}, \mathbf{U}^{0}\right)^{1 / 2}\|\Psi\|_{L^{\infty}\left(\Omega_{T}\right)} .
\end{aligned}
$$

For the first term on the right-hand side of (4.10) we use integration by parts, and $\mathbf{V}^{+}(t, \cdot)=\mathbf{U}_{t}(t, \cdot)$ in $\Omega$, for all $t \in\left[t_{n}, t_{n+1}\right)$,

$$
\begin{gathered}
\int_{0}^{T}\left(\mathbf{V}_{t}, \mathbf{U} \wedge \boldsymbol{\Psi}\right)_{h} \mathrm{~d} t=-\int_{0}^{T}\left(\mathbf{V},[\mathbf{U} \wedge \boldsymbol{\Psi}]_{t}\right)_{h} \mathrm{~d} t-\left(\mathbf{V}^{0}, \mathbf{U}(0, \cdot) \wedge \boldsymbol{\psi}(0, \cdot)\right)_{h} \\
=\int_{0}^{T}\left(-\left[\mathbf{V}-\mathbf{V}^{+}\right]-\mathbf{U}_{t},[\mathbf{U} \wedge \boldsymbol{\Psi}]_{t}\right)_{h} \mathrm{~d} t-\left(\mathbf{V}^{0}, \mathbf{U}(0, \cdot) \wedge \boldsymbol{\psi}(0, \cdot)\right)_{h}
\end{gathered}
$$

Putting things together then yields the assertion of the lemma. 
Effects like numerical integration, interpolation, and combination of successive iterates in (4.9) are considered next to establish convergence of iterates of Algorithm $\mathrm{B}$ to weak solutions of (1.5)-(1.7).

Theorem 4.1. Let the assumptions of Lemma 4.1 be valid, and $\mathbf{U}^{0} \rightarrow \mathbf{u}_{0} \in$ $W^{1,2}\left(\Omega, \mathbb{R}^{m}\right)$, respectively, $\mathbf{V}^{0} \rightarrow \mathbf{v}_{0} \in L^{2}\left(\Omega, \mathbb{R}^{m}\right)$, for $h \rightarrow 0$. There exist $\mathbf{u} \in$ $L^{\infty}\left(0, T, W^{1,2}\left(\Omega ; \mathbb{R}^{m}\right)\right) \cap W^{1, \infty}\left(0, T ; L^{2}\left(\Omega, \mathbb{R}^{m}\right)\right)$, and a subsequence $\left\{\mathbf{U}_{k, h}\right\}$ such that for $(k, h) \rightarrow 0$,

$$
\begin{aligned}
\mathbf{U}_{k, h} & \stackrel{*}{\rightarrow} \mathbf{u} \quad \text { in } L^{\infty}\left(0, T ; W^{1,2}\left(\Omega, \mathbb{R}^{m}\right)\right), \\
\left(\mathbf{U}_{k, h}\right)_{t} & \stackrel{*}{*} \mathbf{u}_{t} \quad \text { in } L^{\infty}\left(0, T ; L^{2}\left(\Omega, \mathbb{R}^{m}\right)\right) .
\end{aligned}
$$

Moreover, $\mathbf{u}: \Omega_{T} \rightarrow \mathbb{R}^{m}$ is a weak solution of (1.5) (1.7).

Throughout the proof, we again drop subindices.

Proof. Step 1. The bounds of Lemma 4.1 yield the existence of $\mathbf{u} \in$ $L^{\infty}\left(0, T ; W^{1,2}\left(\Omega, \mathbb{R}^{m}\right)\right) \cap W^{1, \infty}\left(0, T ; L^{2}\left(\Omega, \mathbb{R}^{m}\right)\right)$, such that for $k \leq \tilde{C} h^{\max \{d / 2,1\}}$ and $h \rightarrow 0$,

$$
\begin{aligned}
& \mathbf{U}, \mathbf{U}^{+}, \overline{\mathbf{U}} \quad \stackrel{*}{\rightarrow} \quad \mathbf{u} \quad \text { in } L^{\infty}\left(0, T ; W^{1,2}\left(\Omega, \mathbb{R}^{m}\right)\right), \\
& \mathbf{U}, \mathbf{U}^{+}, \overline{\mathbf{U}} \rightarrow \mathbf{u} \text { in } L^{2}\left(\Omega_{T}, \mathbb{R}^{m}\right) \text {, } \\
& \mathbf{U}_{t}, \mathbf{V}, \mathbf{V}^{+} \stackrel{*}{\rightarrow} \quad \mathbf{u}_{t} \quad \text { in } L^{\infty}\left(0, T ; L^{2}\left(\Omega, \mathbb{R}^{m}\right)\right) \text {. }
\end{aligned}
$$

Here, the energy identity (4.1) is used to verify convergence of both, $\{\mathbf{V}\}$ and $\left\{\mathbf{V}^{+}\right\}$ towards the same limit as $k, h \rightarrow 0$, since

$$
\begin{aligned}
\left\|\mathbf{V}-\mathbf{V}^{+}\right\|_{L^{2}\left(0, T ; L^{2}\right)}^{2} & \leq C \sum_{n=1}^{N}\left\|\mathbf{V}^{n}-\mathbf{V}^{n-1}\right\|_{h}^{2} \int_{t_{n-1}}^{t_{n}}\left(\frac{s-t_{n}}{k}\right)^{2} \mathrm{~d} s \\
& =C \frac{k^{3}}{3} \sum_{n=1}^{N}\left\|d_{t} \mathbf{V}^{n}\right\|_{h}^{2} \rightarrow 0,
\end{aligned}
$$

and a corresponding result holds for the triple $\{\mathbf{U}\},\left\{\mathbf{U}^{+}\right\}$, and $\{\overline{\mathbf{U}}\}$.

Since $\left|\mathbf{U}^{+}\right|(\mathbf{z})=1$ for all $\mathbf{z} \in \mathcal{N}_{h}$ and all $t \in[0, T]$, there holds $\mathcal{I}_{h}\left[\left|\mathbf{U}^{+}\right|^{2}\right]=1$ for all $(t, \mathbf{x}) \in \overline{\Omega_{T}}$, and for all $K \in \mathcal{T}_{h}$,

$$
\left\|\left|\mathbf{U}^{+}\right|^{2}-1\right\|_{L^{2}(K)} \leq C h\left\|\nabla \mathbf{U}^{+}\right\|_{L^{2}(K)} .
$$

Consequently, $\left|\mathbf{U}^{+}\right| \rightarrow 1$ almost everywhere in $\Omega_{T}$, and hence $|\mathbf{u}|=1$ almost everywhere.

Step 2. We pass to the limit in each of the terms in (4.9). For the first term, because of the wedge product in it, we only need to show $\lim _{k, h \rightarrow 0}\left(\mathbf{U}_{t}, \mathbf{U} \wedge \boldsymbol{\psi}_{t}\right)_{h}=$ $\left(\mathbf{u}_{t}, \mathbf{u} \wedge \boldsymbol{\psi}_{t}\right)$.

$$
\begin{aligned}
& \left|\left(\mathbf{U}_{t}, \mathbf{U} \wedge \boldsymbol{\Psi}_{t}\right)_{h}-\left(\mathbf{u}_{t}, \mathbf{u} \wedge \boldsymbol{\Psi}_{t}\right)\right| \leq\left|\left(\mathbf{U}_{t}, \boldsymbol{I}_{h}\left[\mathbf{U} \wedge \boldsymbol{\Psi}_{t}\right]\right)_{h}-\left(\mathbf{U}_{t}, \mathcal{I}_{h}\left[\mathbf{U} \wedge \boldsymbol{\Psi}_{t}\right]\right)\right| \\
& \quad+\left|\left(\mathbf{U}_{t}, \boldsymbol{I}_{h}\left[\mathbf{U} \wedge \boldsymbol{\Psi}_{t}\right]-\mathbf{U} \wedge \boldsymbol{\Psi}_{t}\right)\right|+\left|\left(\mathbf{U}_{t},[\mathbf{U}-\mathbf{u}] \wedge \boldsymbol{\Psi}_{t}\right)\right| \\
& \quad+\left|\left(\mathbf{u}_{t}-\mathbf{U}_{t}, \mathbf{u} \wedge \boldsymbol{\Psi}_{t}\right)\right|=: I+\cdots+I V
\end{aligned}
$$

We use properties of $(\cdot, \cdot)_{h}, W^{1,2}(\Omega)$-stability of $\mathcal{I}_{h}$, and $\|\mathbf{U}\|_{L^{\infty}} \leq 1$ to find

$$
I \leq C h\left\|\mathbf{U}_{t}\right\|\left\|\nabla \boldsymbol{I}_{h}\left[\mathbf{U} \wedge \boldsymbol{\Psi}_{t}\right]\right\| \leq C h\left\|\mathbf{U}_{t}\right\|(\|\nabla \mathbf{U}\|+1)\left\|\boldsymbol{\Psi}_{t}\right\|_{W^{1, \infty}} .
$$


The term $I I$ can be bounded correspondingly. Convergence towards zero $(h \rightarrow 0)$ of the terms $I I I$ and $I V$ follows from $\mathbf{U} \rightarrow \mathbf{u}$ in $L^{2}\left(\Omega_{T}, \mathbb{R}^{m}\right)$, and $\mathbf{U}_{t} \rightarrow \mathbf{u}_{t}$ in $L^{2}\left(\Omega_{T}, \mathbb{R}^{m}\right)$. Hence, for $k \leq \tilde{C} h^{\max \{d / 2,1\}}$,

$$
\begin{aligned}
\lim _{k, h \rightarrow 0} & \int_{0}^{T}\left(\mathbf{U}_{t}, \mathbf{U} \wedge \boldsymbol{\Psi}_{t}\right)_{h} \mathrm{~d} t \\
& =\int_{0}^{T}\left(\mathbf{u}_{t}, \mathbf{u} \wedge \boldsymbol{\Psi}_{t}\right) \mathrm{d} t \quad \forall \boldsymbol{\Psi} \in C_{0}^{\infty}\left([0, T) ; C^{\infty}\left(\Omega, \mathbb{R}^{m}\right)\right) .
\end{aligned}
$$

Next, we verify that the limit for the second term in (4.9) for $k \leq \tilde{C} h^{\max \{d / 2,1\}}$ is

$$
\begin{aligned}
\lim _{k, h \rightarrow 0} & \int_{0}^{T}\left(\nabla \overline{\mathbf{U}}, \nabla \mathcal{I}_{h}[\overline{\mathbf{U}} \wedge \boldsymbol{\Psi}]\right) \mathrm{d} t \\
& =\int_{0}^{T}(\nabla \mathbf{u}, \nabla[\mathbf{u} \wedge \boldsymbol{\Psi}]) \mathrm{d} t \quad \forall \boldsymbol{\Psi} \in C_{0}^{\infty}\left([0, T) ; C^{\infty}\left(\Omega, \mathbb{R}^{m}\right)\right) .
\end{aligned}
$$

For this purpose, since $\langle\nabla \overline{\mathbf{U}}, \nabla[\overline{\mathbf{U}} \wedge \boldsymbol{\Psi}]\rangle=\langle\nabla \overline{\mathbf{U}}, \overline{\mathbf{U}} \wedge \nabla \boldsymbol{\Psi}\rangle$, and $\langle\nabla \mathbf{u}, \nabla[\mathbf{u} \wedge \boldsymbol{\Psi}]\rangle=$ $\langle\nabla \mathbf{u}, \mathbf{u} \wedge \nabla \boldsymbol{\Psi}\rangle$ almost everywhere,

$$
\begin{gathered}
\left(\nabla \overline{\mathbf{U}}, \nabla \boldsymbol{I}_{h}[\overline{\mathbf{U}} \wedge \boldsymbol{\Psi}]\right)-(\nabla \mathbf{u}, \nabla[\mathbf{u} \wedge \boldsymbol{\Psi}])=\left(\nabla \overline{\mathbf{U}}, \nabla\left\{\mathcal{I}_{h}[\overline{\mathbf{U}} \wedge \boldsymbol{\Psi}]-\overline{\mathbf{U}} \wedge \boldsymbol{\Psi}\right\}\right) \\
+(\nabla \overline{\mathbf{U}},[\overline{\mathbf{U}}-\mathbf{u}] \wedge \nabla \boldsymbol{\Psi})+(\nabla[\overline{\mathbf{U}}-\mathbf{u}], \mathbf{u} \wedge \nabla \boldsymbol{\Psi})=: I+I I+I I I
\end{gathered}
$$

We compute $I \leq C h\|\nabla \overline{\mathbf{U}}\|(\|\nabla \overline{\mathbf{U}}\|+1)\|\Psi\|_{W^{2, \infty}}$, by an interpolation estimate, using $\left.D^{2} \overline{\mathbf{U}}\right|_{K}=0$ for all $K \in \mathcal{T}_{h}$. For the terms $I I$, respectively, $I I I$, we use $\overline{\mathbf{U}} \rightarrow \mathbf{u}$ in $L^{2}\left(\Omega_{T}, \mathbb{R}^{m}\right)$, and $\nabla \mathbf{U} \rightarrow \nabla \mathbf{u}$ in $L^{2}\left(\Omega_{T}, \mathbb{R}^{m d}\right)$, respectively, to conclude that $\int_{0}^{T} I I \mathrm{~d} t, \int_{0}^{T} I I I \mathrm{~d} t \rightarrow 0$, for $k \leq \tilde{C} h^{\max \{d / 2,1\}}$, and $h \rightarrow 0$. Therefore, assertion (4.15) is valid.

Convergence $\left(\mathbf{V}^{0}, \overline{\mathbf{U}}(0, \cdot) \wedge \boldsymbol{\Psi}(0, \cdot)\right)_{h} \rightarrow\left(\mathbf{v}_{0}, \mathbf{u}_{0} \wedge \boldsymbol{\Psi}(0, \cdot)\right)$, for $k \leq \tilde{C} h^{\max \{d / 2,1\}}$, and $h \rightarrow 0$ follows from properties of $(\cdot, \cdot)_{h}$, and $\mathbf{V}^{0} \rightarrow \mathbf{v}_{0}$ in $L^{2}\left(\Omega, \mathbb{R}^{m}\right)$, respectively, $\mathbf{U}^{0} \rightarrow \mathbf{u}_{0}$ in $W^{1,2}\left(\Omega, \mathbb{R}^{m}\right)$. Finally, since $|\mathbf{U}| \leq 1$, we conclude

$$
\begin{aligned}
\int_{0}^{T} & \left(\mathbf{V}^{+}-\mathbf{V},[\mathbf{U} \wedge \boldsymbol{\Psi}]_{t}\right)_{h} \mathrm{~d} t \\
& \leq C k^{1 / 2}\left(k \int_{0}^{T}\left\|\mathbf{V}_{t}\right\|^{2} \mathrm{~d} t\right)^{1 / 2}\left(1+\int_{0}^{T}\left\|\mathbf{U}_{t}\right\|^{2} \mathrm{~d} t\right)^{1 / 2}\left(1+\|\boldsymbol{\Psi}\|_{W^{1, \infty}\left(\Omega_{T}\right)}\right),
\end{aligned}
$$

for all but one term in (4.9). Therefore, $\mathbf{u}: \Omega_{T} \rightarrow \mathbb{R}^{m}$ satisfies assertion (iii) of Definition 4.1 .

Step 3. We verify assertion (iv) of Definition 4.1, $\mathbf{u}_{0}=\lim _{t \rightarrow 0} \lim _{k, h \rightarrow 0} \mathbf{U}(t, \cdot)$ in $L^{2}\left(\Omega, \mathbb{R}^{m}\right)$ follows from (4.12). It remains to show $\mathbf{U}_{t}(t, \cdot) \rightarrow \mathbf{v}_{0}$ in $L^{2}\left(\Omega, \mathbb{R}^{m}\right)$ as $t \rightarrow 0$. Therefore, multiply $\left(\mathbf{u}_{t t}-\Delta \mathbf{u}\right) \wedge \mathbf{u}=0$ with $\boldsymbol{\Psi} \in C_{0}^{\infty}\left([0, T) ; C^{\infty}\left(\Omega, \mathbb{R}^{m}\right)\right)$, integrate by parts on $\Omega_{T}$, and subtract the resulting equation from (4.9). We find for the limit $k, h \rightarrow 0$,

$$
\left(\mathbf{u}_{t}(0, \cdot)-\mathbf{v}_{0}, \mathbf{u}_{0} \wedge \boldsymbol{\Psi}\right)=0 \quad \forall \boldsymbol{\Psi} \in C_{0}^{\infty}\left([0, T) ; C^{\infty}\left(\Omega, \mathbb{R}^{m}\right)\right) .
$$

On noting $\left\langle\mathbf{v}_{0}(\mathbf{x}), \mathbf{u}_{0}(\mathbf{x})\right\rangle=\left\langle\mathbf{u}_{t}(0, \mathbf{x}), \mathbf{u}_{0}(\mathbf{x})\right\rangle=0$ for almost every $\mathbf{x} \in \Omega$, it follows from the vector identity $\mathbf{v}=\langle\mathbf{u}, \mathbf{v}\rangle \mathbf{u}-\mathbf{u} \wedge(\mathbf{u} \wedge \mathbf{v})$ - with $\mathbf{v}=\mathbf{u}_{t}(0, \cdot)-\mathbf{v}_{0}$ and $\mathbf{u}=\mathbf{u}_{0}$ - that $\mathbf{u}_{t}(t, \cdot) \rightarrow \mathbf{v}_{0}$ in $L^{2}\left(\Omega, \mathbb{R}^{m}\right)$ as $t \rightarrow 0$. 
We also need to show $\mathbf{u}_{t}(t, \cdot) \rightarrow \mathbf{v}_{0}$ in $L^{2}\left(\Omega, \mathbb{R}^{m}\right)$ as $t \rightarrow 0$. By weak lower semicontinuity of $L^{2}$-norm and Fatou's lemma

$$
\|D \mathbf{u}(t, \cdot)\|_{L^{2}} \leq \liminf _{k, h \rightarrow 0}\|D \mathbf{U}(t, \cdot)\|_{L^{2}} \quad t \geq 0 .
$$

Hence, for all $t \geq 0$, because of properties of $(\cdot, \cdot)_{h}$, and assumptions on initial data,

$$
\begin{aligned}
E\left(\mathbf{u}_{t}(t, \cdot), \mathbf{u}(t, \cdot)\right) & \leq \liminf _{k, h \rightarrow 0} E\left(\mathbf{U}_{t}(t, \cdot), \mathbf{U}(t, \cdot)\right) \\
& =\liminf _{k, h \rightarrow 0} E_{h}\left(\mathbf{U}_{t}(t, \cdot), \mathbf{U}(t, \cdot)\right) \leq E\left(\mathbf{v}_{0}, \mathbf{u}_{0}\right) .
\end{aligned}
$$

Therefore,

$$
\limsup _{t \rightarrow 0}\left\|\mathbf{u}_{t}(t, \cdot)\right\|_{L^{2}} \leq\left\|\mathbf{v}_{0}\right\|_{L^{2}}, \quad \limsup _{t \rightarrow 0}\|\nabla \mathbf{u}(t, \cdot)\|_{L^{2}} \leq\left\|\nabla \mathbf{u}_{0}\right\|_{L^{2}},
$$

and the weak convergence $\mathbf{u}_{t}(t, \cdot) \rightarrow \mathbf{v}_{0}$ in $L^{2}\left(\Omega, \mathbb{R}^{m}\right)$ and $\nabla \mathbf{u}(t, \cdot) \rightarrow \nabla \mathbf{u}_{0}$ in $L^{2}\left(\Omega, \mathbb{R}^{m d}\right)$ implies strong convergence $\mathbf{u}_{t}(t, \cdot) \rightarrow \mathbf{v}_{0}$ and $\nabla \mathbf{u}(t, \cdot) \rightarrow \nabla \mathbf{u}_{0}$ in $L^{2}\left(\Omega, \mathbb{R}^{m d}\right)$ as $t \rightarrow 0$. Consequently, $\mathbf{u}: \Omega_{T} \rightarrow \mathbb{R}^{m}$ attains prescribed initial data continuously in $W^{1,2}\left(\Omega, \mathbb{R}^{m}\right) \times L^{2}\left(\Omega, \mathbb{R}^{m}\right)$.

Since all requirements of Definition 4.1 are verified, hence, the map $\mathbf{u}: \Omega_{T} \rightarrow \mathbb{R}^{m}$ is a weak solution to (1.5) (1.7). The proof is complete.

Remark 4.1. A symmetric variant of Algorithm B is: For $n=1,2, \ldots$, find $\left(\mathbf{U}^{n+1}, \lambda^{n}\right)$ $\in \mathbf{V}_{h} \times V_{h}$, such that for all $\mathbf{\Phi} \in \mathbf{V}_{h}$, and all $\mathbf{z} \in \mathcal{N}_{h}$, there holds

$$
\begin{aligned}
& \left(d_{t}^{2} \mathbf{U}^{n+1}, \boldsymbol{\Phi}\right)_{h}+\left(\frac{1}{2} \nabla\left[\mathbf{U}^{n+1}+\mathbf{U}^{n-1}\right], \nabla \boldsymbol{\Phi}\right)=\left(\frac{\lambda^{n}}{2}\left[\mathbf{U}^{n+1}+\mathbf{U}^{n-1}\right], \boldsymbol{\Phi}\right)_{h}, \\
& \lambda^{n}(\mathbf{z})= \begin{cases}0 & \text { for }\left[\mathbf{U}^{n+1}+\mathbf{U}^{n-1}\right](z) \\
\frac{-\left\langle d_{t} \mathbf{U}^{n}(\mathbf{z}), d_{t} \mathbf{U}^{n+1}(\mathbf{z})\right\rangle}{\left|\frac{1}{2}\left[\mathbf{U}^{n+1}+\mathbf{U}^{n-1}\right](\mathbf{z})\right|^{2}} & \\
+\frac{1}{4} \frac{\left(\nabla\left[\mathbf{U}^{n+1}+\mathbf{U}^{n-1}\right],\left[\mathbf{U}^{n+1}+\mathbf{U}^{n-1}\right](\mathbf{z}) \otimes \nabla \varphi_{\mathbf{z}}\right)}{\beta_{\mathbf{z}}\left|\frac{1}{2}\left[\mathbf{U}^{n+1}+\mathbf{U}^{n-1}\right](\mathbf{z})\right|^{2}} & \text { else. }\end{cases}
\end{aligned}
$$

This choice of $\lambda^{n}$ again ensures that $\left|\mathbf{U}^{n+1}(\mathbf{z})\right|=1$ for all $\mathbf{z} \in \mathcal{N}_{h}$. The discrete energy is denoted as $\tilde{E}_{h}\left(\mathbf{V}^{n},\left\{\mathbf{U}^{n-j}\right\}_{j=0}^{1}\right)=\frac{1}{2}\left[\left\|\mathbf{V}^{n}\right\|_{h}^{2}+\frac{1}{2}\left(\left\|\nabla \mathbf{U}^{n}\right\|^{2}+\left\|\nabla \mathbf{U}^{n-1}\right\|^{2}\right)\right]$, for $\mathbf{V}^{n}:=d_{t} \mathbf{U}^{n}$. Again, existence of solutions $\mathbf{U}^{n+1} \in \mathbf{V}_{h}$ in the case $k \leq$ $\tilde{C} h^{\max \{d / 2,1\}}$ can be shown, and

$$
\tilde{E}_{h}\left(\mathbf{V}^{N+1},\left\{\mathbf{U}^{N+1-j}\right\}_{j=0}^{1}\right)=\frac{1}{2}\left[\left\|\mathbf{V}^{0}\right\|_{h}^{2}+\frac{1}{2}\left(\left\|\nabla \mathbf{U}^{1}\right\|^{2}+\left\|\nabla \mathbf{U}^{0}\right\|^{2}\right)\right] \quad(N \geq 0) .
$$

However, convergence for $(k, h) \rightarrow 0$ is not clear because of the absence of the second term on the left-hand side of (4.1), which gives enough control over temporal variations of $\left\{d_{t} \mathbf{U}^{n}\right\}$ to pass to the limit $(k, h) \rightarrow 0$ in every term in (B).

\section{Computational studies}

In this section, we report on the practical performance of Algorithms A and B. The nonlinear systems of equations in each time step were approximately solved using fixed-point iterations (which utilize the old $\lambda^{n+1, \ell}$ defined through the actual iterate $\mathbf{U}^{n+1, \ell}$ to determine the update $\mathbf{U}^{n+1, \ell+1}$ ). Both algorithms were implemented in MATLAB with a direct solution of linear systems of equations. The initial data that we employ for both, the harmonic map heat flow as well as the wave map evolution, in the following two subsections are defined in the following example. 
Example 5.1. Given $w>0$, let $\Omega:=(0,2+w) \times(0,1)$. With $r_{j} \equiv r_{j}(\mathbf{x}):=\left|\mathbf{x}-\mathbf{p}_{j}\right|$ and $a_{j}=a_{j}(\mathbf{x}):=\left(1-2 r_{j}(\mathbf{x})\right)^{4}$ for $\mathbf{x} \in \Omega, j=1,2$, and $\mathbf{p}_{1}:=(1 / 2,1 / 2)$ and $\mathbf{p}_{2}:=(3 / 2,1 / 2)$, we define for $\mathbf{x}=\left(x_{1}, x_{2}\right) \in \Omega$,

$$
\mathbf{u}_{0}(\mathbf{x}):=\left\{\begin{array}{l}
(0,0,-1), \quad x_{1} \in(0,1) \text { and } r_{1} \geq 1 / 2, \\
\frac{\left(2\left(x_{1}-1 / 2\right) a_{1}, 2\left(x_{2}-1 / 2\right) a_{1}, a_{1}^{2}-r_{1}^{2}\right)}{a_{1}^{2}+r_{1}^{2}}, \quad x_{1} \in(0,1) \text { and } r_{1} \leq 1 / 2, \\
\left(0, \sin \left(\left(x_{1}-1\right) \pi / w\right), \cos \left(\left(x_{1}-1\right) \pi / w\right)\right), \quad x_{1} \in(1,1+w) \\
(0,0,1), \quad x_{1} \in(1+w, 2+w) \text { and } r_{2} \geq 1 / 2, \\
\frac{\left(2\left(x_{1}-1 / 2\right) a_{2}, 2\left(x_{2}-1 / 2\right) a_{2}, a_{2}^{2}-r_{2}^{2}\right)}{a_{1}^{2}+r_{1}^{2}}, \quad x_{1} \in(1+w, 2+w) \text { and } r_{2} \leq 1 / 2 .
\end{array}\right.
$$

A projection of the nodal interpolant of the vector field $\mathbf{u}_{0}$ onto the $x y$-plane is shown in the top plot of Figure 5. For the simulation of the wave flow we also define the initial velocity $\mathbf{v}_{0}:=\mathbf{0}$. All employed triangulations were obtained from uniform refinements of the triangulation $\mathcal{T}_{0}$ of $\Omega$ which consists of 6 triangles which are all halved squares if $w=1$. The discrete initial data is obtained by nodal interpolation of $\mathbf{u}_{0}$. Unless otherwise stated, we set $w=1$.

5.1. Experimental results for the wave map problem. We run Algorithm B for Example 5.1 with the triangulation $\mathcal{T}_{3}$ obtained from three uniform ("red-") refinements of $\mathcal{T}_{0}$ and with $k=h / 8$, where $h=2^{-3}$. We stopped the time stepping at $t=1 / 4$, i.e., after 32 time steps, and replaced $\mathbf{V}(t, \cdot)$ by $-\mathbf{V}(t, \cdot)$ at $t=1 / 4$ to reverse the evolution and run another 32 time steps. The numerical results for $t=1 / 4$ and the almost recovered initial data are shown in the second and third (from top) plot of Figure 5. Owing to an instability related to occurrence of large (maximal) gradients which motivates finite-time blow-up, we cannot (approximately) recover the initial data when we reverse the evolution at $t=1 / 2$, which is beyond the instability; cf. the fourth and fifth plots in Figure 5. This behavior does not improve when we significantly decrease the stopping criterion for the fixed point iteration, i.e., when we solve the nonlinear systems of equations almost exactly.

In order to compare the performance of Algorithm B to the projection strategy proposed in [7] we display in Figure 1 the total energy of the approximations obtained with the two schemes on uniform triangulations with mesh-size $h=2^{-3}, 2^{-4}$, and for time-step sizes $k=h, h / 10$. We recall that Algorithm 3.1 in [7] involves a projection step. In particular, given $\left(\mathbf{U}^{j}, \mathbf{V}^{j}\right) \in \mathbf{V}_{h}^{2}$ satisfying $\left|\mathbf{U}^{j}(\mathbf{z})\right|=1$ and for all nodes $\mathbf{z}$ each iteration of that algorithm performs the following two steps:

(1) Compute $\mathbf{V}^{j+1} \in \mathbf{V}_{h}$ such that $\mathbf{U}^{j}(\mathbf{z}) \cdot \mathbf{V}^{j+1}(\mathbf{z})=0$ for all $\mathbf{z} \in \mathcal{N}_{h}$ and

$$
\left(d_{t} \mathbf{V}^{j+1}, \boldsymbol{\Phi}\right)_{h}=\left(\nabla \mathbf{U}^{j}, \nabla \boldsymbol{\Phi}\right) \quad \forall \boldsymbol{\Phi} \in \mathbf{V}_{h}
$$

Define $\mathbf{U}^{j+1} \in \mathbf{V}_{h}$ through

$$
\mathbf{U}^{j+1}(\mathbf{z})=\frac{\mathbf{U}^{j+1}(\mathbf{z})+k \mathbf{V}^{j+1}(\mathbf{z})}{\left|\mathbf{U}^{j+1}(\mathbf{z})+k \mathbf{V}^{j+1}(\mathbf{z})\right|} \text { for all } \mathbf{z} \in \mathcal{N}_{h} .
$$

We observe that the total energy is for all pairs of discretization parameters decreasing for the numerical approximation obtained with Algorithm B. This is not the case for the approximations computed with the explicit projection scheme of [7; in fact, the energy rapidly increases for $k=h$ which indicates strong numerical instabilities. Nevertheless, for $k=h / 10$ all results are qualitatively comparable. The total energy, the kinetic energy $E_{h}^{\text {kin }}(\mathbf{V}(t, \cdot)):=\frac{1}{2}|| \mathbf{V}(t, \cdot) \|_{h}^{2}$, and the $W^{1, \infty}(\Omega)$ semi-norm as functions of time $t \in(0,1 / 2)$ for the two different schemes on a 
triangulation with $h=2^{-5}$ and $k=h / 10$ are displayed in Figure 2. We observe that large gradients occur and that energy is lost when a large change of the $W^{1, \infty}(\Omega)$ norms takes place.

The experimental results are slightly different when the symmetric but theoretically unjustified scheme of Remark 4.1 is used to compute numerical approximations. As opposed to Algorithm B and the projection scheme of [7, the evolution can always be reversed. The reported irreversibility in Example 5.1 which is related to a numerical instability when the vectors at $(1 / 2,1 / 2)$ or $(3 / 2,1 / 2)$ changes its direction within a small time interval is different here and the vectors remain fixed when the symmetric scheme is used. Also, there is no loss of (the modified) energy as can be seen in Figure 3. Nevertheless, we emphasize that the symmetric scheme is not known to converge to a weak solution.

5.2. Experimental results for the heat flow problem. Our numerical experiments for the harmonic map heat flow problem based on Algorithm A do not show significant advantages of the proposed scheme over the algorithms developed in 6. 3. The reason for this is that the fixed point iteration requires in all of our numerical studies that $k \leq h^{2} / 5$, and therefore does not improve existing results. However, for this choice of the time-step size we obtain reasonable results for the evolution defined by the initial data specified in Example 5.1. Figure 6 displays snapshots of the numerical solution for $t=0,0.01875,00375,0.05625,0.075$. Large (maximal) gradients occur for $t \approx 0.05$ and afterwards the solution appears to be smooth and converges to a steady (uniformly constant) state. We remark that the numerical results do not change qualitatively when we employ other values for the parameter $w$. Rapid decay of the energy accompanied by occurrence of large gradients on each mesh when $k \leq h^{2} / 5$ are the main conclusions of the practical experience with Algorithm A.

5.3. Effect of different winding numbers. As is detailed in [14, weak solutions of the Dirichlet problem for the harmonic map heat flow for $\ell$-equivariant maps, with $\ell=2$, do not blow up. This is in contrast to the wave-map flow, where finitetime blow-up behavior is still expected; cf. [18, Remark 1.6]. The following example reports on corresponding numerical studies in an equivariant setting but restricted to a square centered around the origin. The initial data that we use are defined as follows.

Example 5.2. Set $\Omega:=(-2,2)^{2}$ and for $\ell=1$ or $\ell=2$ define

$$
\mathbf{u}_{0}(r, \theta):=\left(\begin{array}{c}
\sin \chi(r, \ell \theta) \sin \ell \theta \\
\sin \chi(r, \ell \theta) \cos \ell \theta \\
\cos \chi(r, \ell \theta)
\end{array}\right) \quad \mathbf{v}_{0}(r, \theta) \equiv 0,
$$

where $(r, \theta)$ denotes polar coordinates in $\mathbb{R}^{2}$ and $\chi(r, \ell \theta):=\left(r^{3} / 4\right) \exp (-(4(r-$ 2)/10 $\left.)^{4}\right)$.

We ran Algorithm B in Example 5.2 on uniform triangulations of $\Omega$ with $h=$ $4 \cdot 2^{-5}$ and $h=4 \cdot 2^{-6}$ and time-step size defined through $k=h / 5$. Figure 7 displays the total energies and $W^{1, \infty}$ semi-norm as functions of $t \in[0,4]$. We observe that large gradients occur for $\ell=1$ while they do not occur for $\ell=2$. 


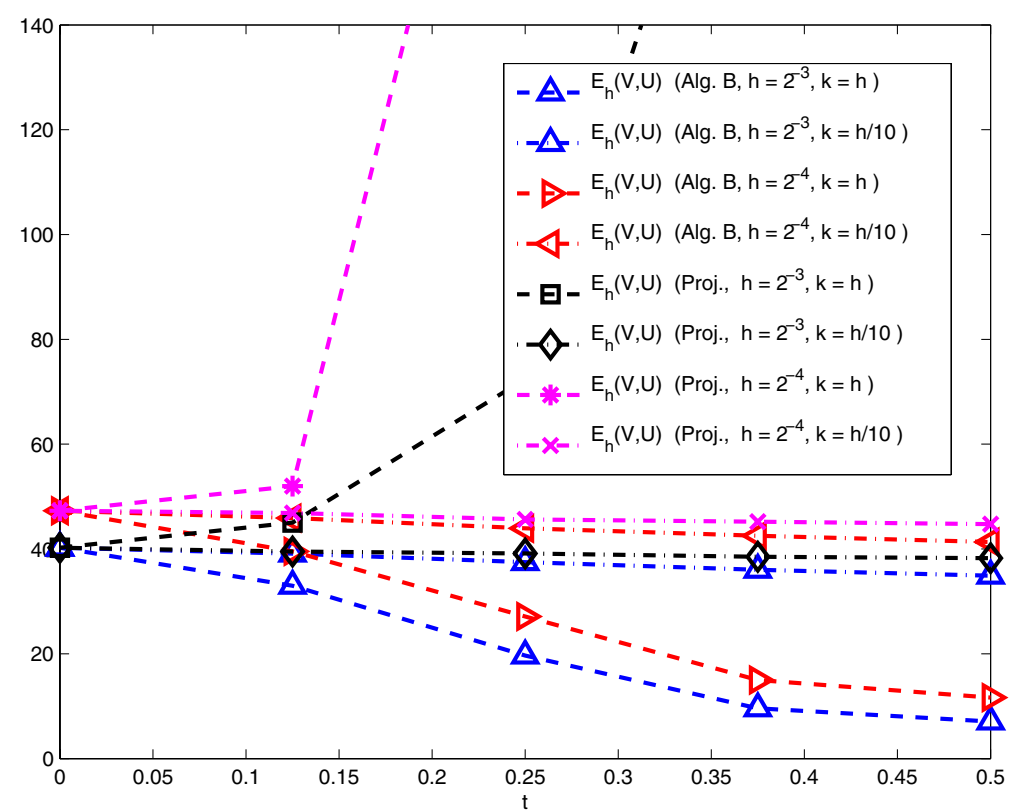

Figure 1. Total energy for numerical approximations obtained with Algorithm B and with the projection scheme of 7 for various discretization parameters in the wave map problem defined with initial data from Example 5.1 .

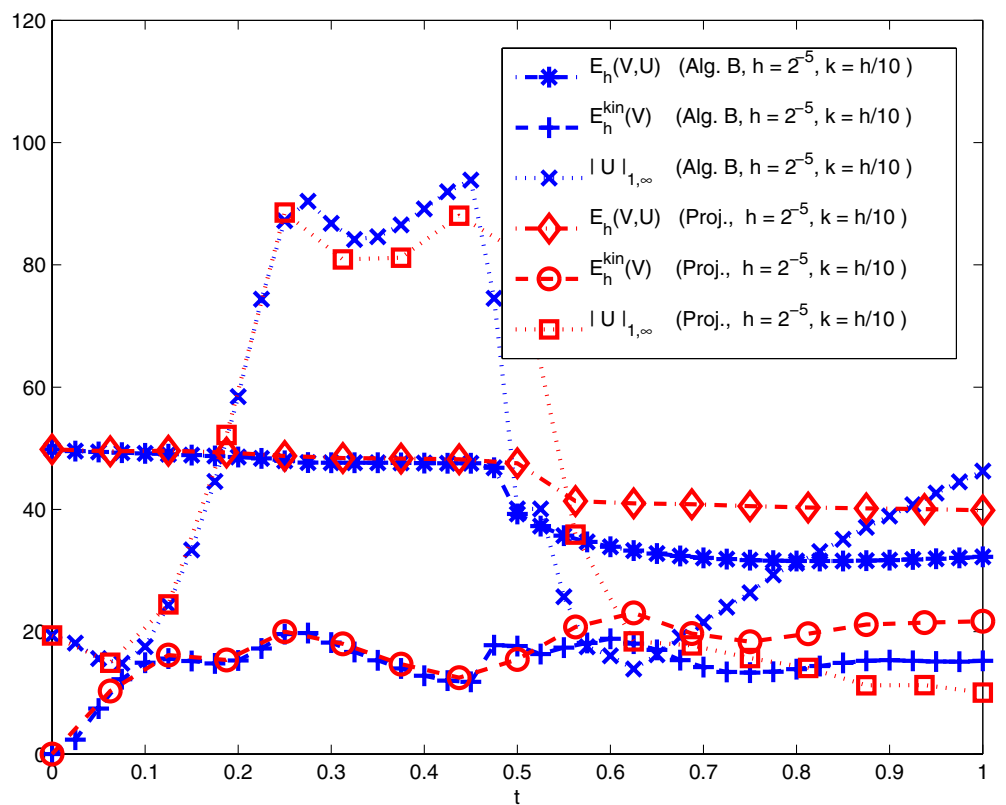

Figure 2. Total energy, kinetic energy, and $W^{1, \infty}(\Omega)$-semi-norm for numerical approximations obtained with Algorithm B and with the projection scheme of [7] for fixed discretization parameters in the wave map problem defined with initial data from Example 5.1. 


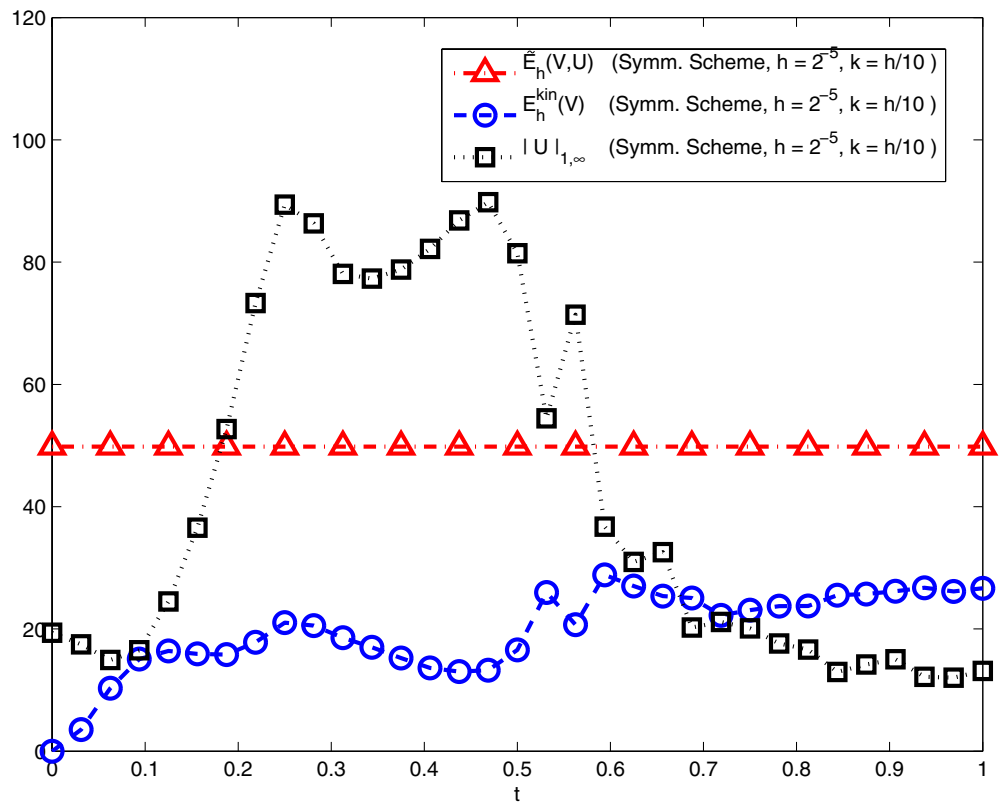

Figure 3. Total energy, kinetic energy, and $W^{1, \infty}(\Omega)$-semi-norm for numerical approximations obtained with the symmetric scheme from Remark 4.1 for fixed discretization parameters in the wave map problem defined with initial data from Example 5.1 .

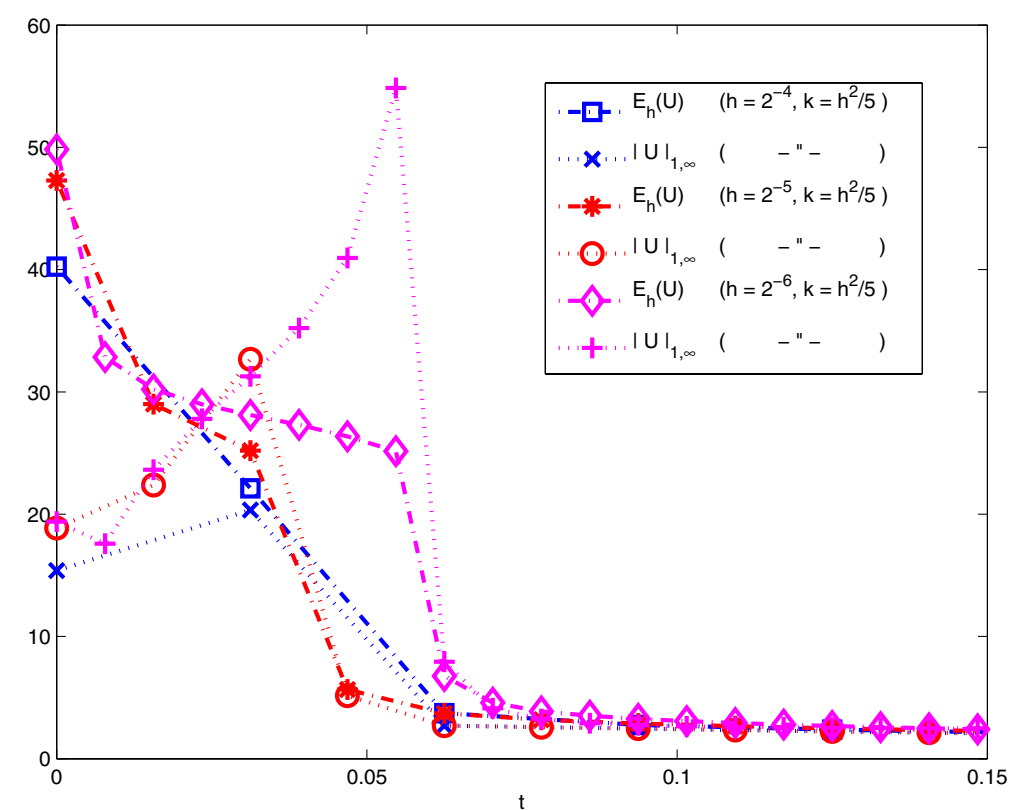

Figure 4. Energy and $W^{1, \infty}$ semi-norm for numerical approximations of the harmonic map heat flow problem obtained with Algorithm A for various discretizations parameters and with initial data defined in Example 5.1 . 

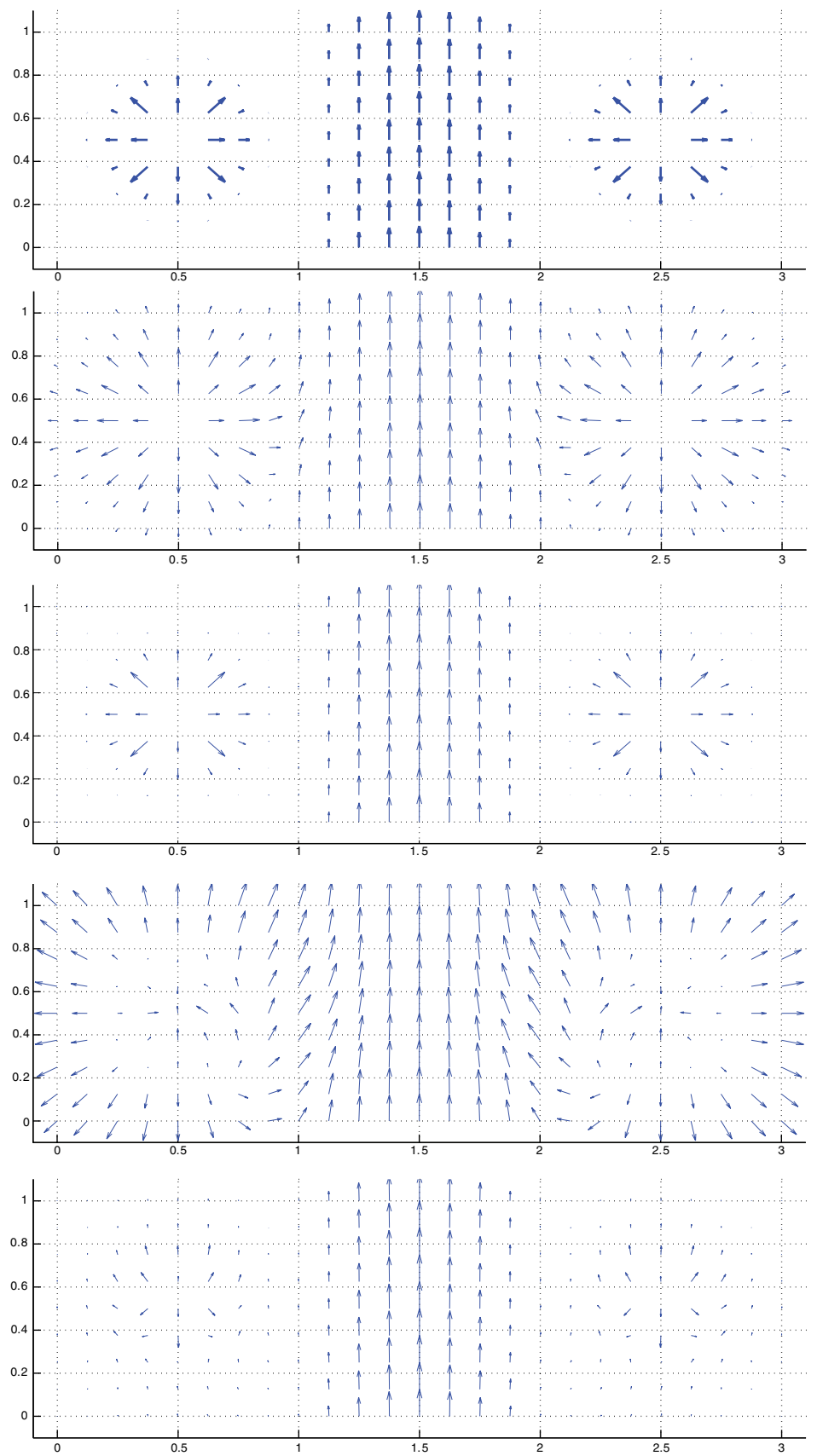

FiguRE 5. Numerical solutions obtained with Algorihm B in Example 5.1. Initial data (top plot), numerical approximations at $t=1 / 4$ and $t=1 / 2$ (second and fourth plots from top), and approximations at $t=0$ when the evolution is reversed at $t=1 / 4$ and $t=1 / 2$ (third and fifth plots). All vectors are scaled by the factor $1 / 8$ for graphical purposes. 

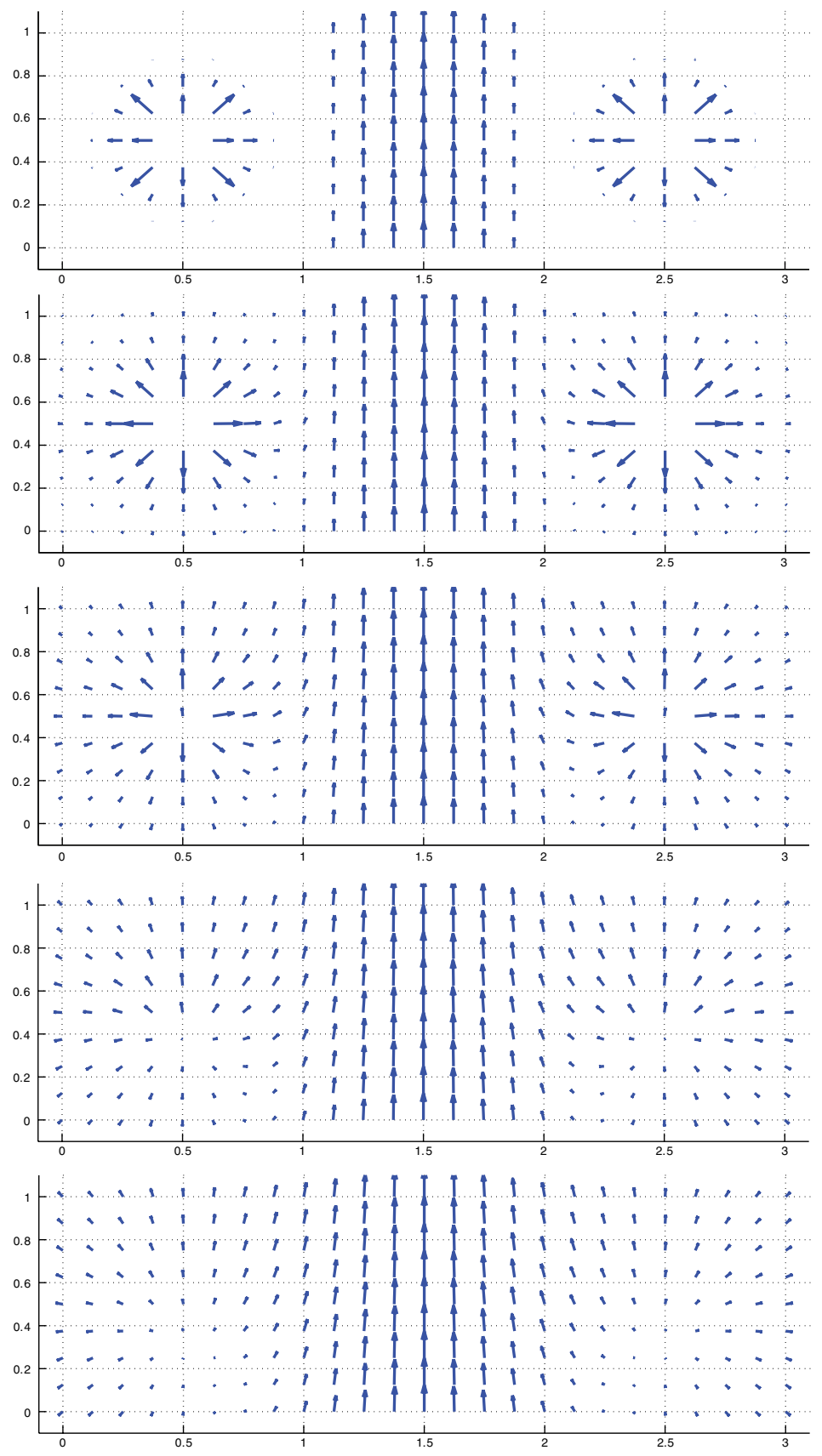

FiguRE 6. Snapshots of the numerical solutions for evolution governed by harmonic map heat flow in Example 5.1 and simulated with Algorithm A. Displayed solutions correspond to $t=$ $0,0.01875,00375,0.05625,0.075$ (from top to bottom). All vectors are scaled by the factor $1 / 8$ for graphical purposes. 


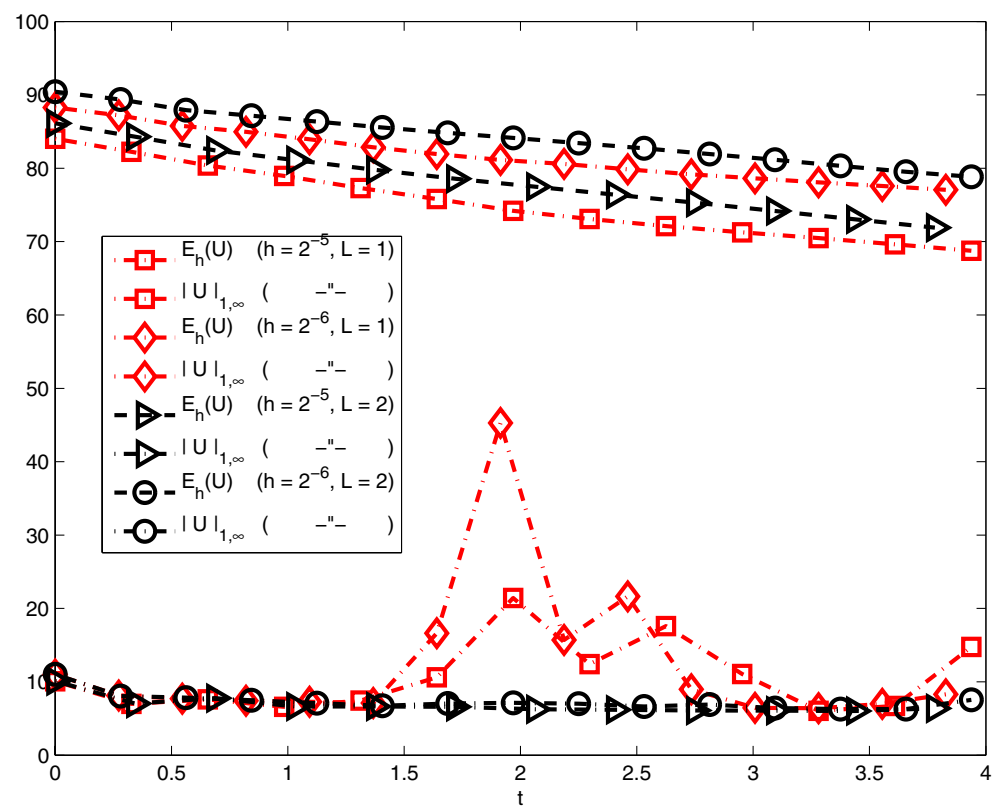

FiguRE 7. Energy and $W^{1, \infty}$ semi-norm for numerical approximations of the wave map problem obtained with Algorithm B for various discretizations parameters and with initial data defined in Example 5.2 .

\section{ACKNOWLEDGMENT}

The first author (S.B.) was supported by Deutsche Forschungsgemeinschaft through the DFG Research Center MATHEON 'Mathematics for key technologies' in Berlin.

\section{REFERENCES}

[1] F. Alouges, A new algorithm for computing liquid crystal stable configurations: the harmonic mapping case, SIAM J. Numer. Anal. 34, pp. 1708-1726 (1997). MR1472192 (98k:82190)

[2] F. Alouges, P. Jaisson Convergence of a finite elements discretization for the Landau Lifshitz equations, Math. Models Methods Appl. Sci. 16, pp. 299-316 (2006). MR 2210092 (2007b:65091)

[3] S. Bartels, A. Prohl, Constraint preserving implicit finite element discretization of harmonic map flow into spheres, Math. Comp. 76, pp. 1847-1859 (2007). MR2336271 (2008j:65156)

[4] S. Bartels, A. Prohl, Convergence of an implicit finite element method for the Landau-Lifshitz equation, SIAM J. Numer. Anal. 44, pp. 1405-1419 (2006). MR2257110 (2007g:65087)

[5] S. Bartels, A. Prohl, Stable discretization of scalar and constrained vectorial Perona-Malik equation, Interfaces and Free Boundaries 9, pp. 431-453 (2007). MR2358212

[6] J.W. Barrett, S. Bartels, X. Feng, A. Prohl, A convergent and constraint-preserving finite element method for the p-harmonic flow into spheres, SIAM J. Numer. Anal. 45, pp. 905-927 (2007). MR2318794 (2008f:65170)

[7] S. Bartels, X. Feng, A. Prohl, Finite element approximations of wave maps into spheres, SIAM J. Numer. Anal. 46, pp. 61-87 (2007). MR2377255 (2008k:35318)

[8] S. Bartels, A. Prohl, Convergence of an implicit, constraint preserving finite element discretization of p-harmonic heat flow into the sphere, Numer. Math. 109 (2008), no. 4, 489-507. $\operatorname{MR} 2407320$ 
[9] P. Bizoń, T. Chmaj, Z. Tabor, Dispersion and collapse of wave maps, Nonlinearity 13, pp. 1411-1423 (2000). MR1767966 (2001g:58047)

[10] P. Bizon, T. Chmaj, Z. Tabor, Formation of singularities for equivariant $(2+1)$ dimensional wave maps into the 2-sphere, Nonlinearity 14, p. 1041-1053 (2001). MR1862811 (2003b:58043)

[11] K.C. Chang, W.Y. Ding, R. Ye, Finite-time blow-up of the heat flow of harmonic maps from surfaces, J. Diff. Geom. 36, pp. 507-511 (1992). MR1180392 (93h:58043)

[12] Y.M. Chen, M. Struwe, Existence and partial regularity results for the heat flow for harmonic maps, Math. Z. 201, pp. 83-103 (1989). MR990191(90i:58031)

[13] J.-M. Coron, J.-M. Ghidaglia, Explosion en temps fini pour le flot des applications harmoniques, CR. Acad. Sci., Paris, Ser. I 308, pp. 339-344 (1989). MR992088 (90g:58026)

[14] J.F. Grotowski, J. Shatah, A note on geometric heat flows in critical dimensions, Preprint (2006), downloadable at: http://math.nyu.edu/faculty/shatah/preprints/gs06.pdf.

[15] E. Hairer, C. Lubich, G. Wanner, Geometric numerical integration: structure-preserving algorithms for ordinary differential equations, 2nd edition, Springer (2006). MR2221614 (2006m:65006)

[16] J. Krieger, W. Schlag, D. Tataru, Renormalization and blow up for charge one equivariant critical wave maps, Invent. Math. 171 (2008), no. 3, 543-615. MR2372807

[17] M. Kruzik, A. Prohl, Recent developments in the modeling, analysis, and numerics of ferromagnetism, SIAM Review 48, pp. 439-483 (2006). MR2278438(2008c:82088)

[18] I. Rodnianski, J. Sterbenz, On the formation of singularities in the critical $O(3) \sigma$-model, preprint (arXiv-series), (2006).

[19] J. Shatah, Weak solutions and development of singularities in the $S U(2)$ model, Comm. Pure Appl. Math. 41, pp. 459-469 (1988). MR933231 (89f:58044)

[20] J. Shatah, M. Struwe, Geometric wave equations, New York University, Courant Institute of Mathematical Sciences, New York (1998). MR 1674843 (2000i:35135)

[21] R.E. Showalter, Monotone operators in Banach space and nonlinear partial differential equations, AMS (1997). MR1422252 (98c:47076)

[22] M. Struwe, Geometric evolution problems, IAS/Park City Math. Series, vol. 2, pp. 259-339 (1996). MR.1369591 (97e:58057)

[23] M. Struwe, On the evolution of harmonic maps of Riemannian surfaces, Math. Helv. 60, pp. 558-581 (1985) MR826871 (87e:58056)

[24] B. Tang, G. Sapiro, V. Caselles, Diffusion of generated data on non-flat manifolds via harmonic maps theory: the direction diffusion case. Int. J. Comput. Vision 36, pp. 149-161 (2000).

[25] B. Tang, G. Sapiro, V. Caselles, Color image enhancement via chromaticity diffusion, IEEE Trans. Image Proc. 10, pp. 701-707 (2001).

[26] D. Tataru, The wave maps equation, Bull. Amer. Math. Soc. 41, pp. 185-204 (2004). MR2043751 (2005h:35245)

[27] L.A. Vese, S.J. Osher, Numerical methods for p-harmonic flows and applications to image processing, SIAM J. Numer. Anal. 40, pp. 2085-2104 (2002). MR.1974176 (2004k:65102)

Institute for Numerical Simulation, Rheinische Friedrich-Wilhelms-Universität Bonn, Wegelerstrasse 6, D-53115 Bonn, Germany

E-mail address: bartels@ins.uni-bonn.de

Mathematisches Institut, Universität Tübingen, Auf Der Morgenstelle 10, D-72076 Tübingen, Germany

E-mail address: lubich@na.uni-tuebingen.de

Mathematisches Institut, Universität Tübingen, Auf Der Morgenstelle 10, D-72076 Tübingen, Germany

E-mail address: prohl@na.uni-tuebingen.de 